\title{
Data report: quantification of potential drilling contamination using perfluorocarbon tracer at IODP Expedition 329 sites $^{1}$
}

\author{
Justine Sauvage, ${ }^{2}$ Leah Lewis, ${ }^{3}$ Dennis Graham, ${ }^{2}$ Arthur J. Spivack, ${ }^{2}$ and Steven D'Hondt ${ }^{3}$
}

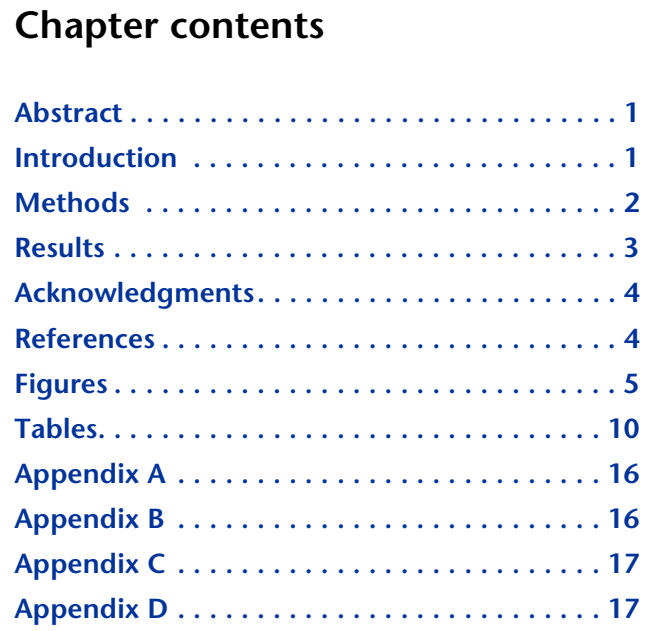

'Sauvage, J., Lewis, L., Graham, D., Spivack, A.J., and D'Hondt, S.D., 2017. Data report:

quantification of potential drilling contamination using perfluorocarbon tracer at IODP Expedition 329 sites. In D'Hondt, S., Inagaki, F., Alvarez Zarikian, C.A., and the Expedition 329 Scientists, Proceedings of the Integrated Ocean Drilling Program, 329: Tokyo (Integrated Ocean Drilling Program Management International, Inc.). doi:10.2204/iodp.proc.329.204.2017

${ }^{2}$ Graduate School of Oceanography, University of Rhode Island, Narragansett RI 02882, USA.

Correspondence author: Justine_sauvage@my.uri.edu

${ }^{3}$ Scripps Institution of Oceanography, University of California San Diego, La Jolla CA 92037, USA.

\section{Abstract}

To quantify the potential for biological contamination associated with the coring process, we conducted perfluorocarbon tracer (PFT) analysis on 556 sediment samples from Integrated Ocean Drilling Program (IODP) Expedition 329. The expedition cored deep-sea sediment at seven sites in the South Pacific Gyre (Sites U1365-U1371). We analyzed two types of sediment samples: (1) samples taken in the central part of the core (i.e., interior samples) and (2) samples taken near the core edge (i.e., exterior samples). We calculated the amount of potential drilling fluid intrusion from the mass of PFT that we measured in each sample. For the seven Expedition 329 sites (15 holes analyzed), PFT content ranges from below detection to levels where contamination is extremely apparent. The centers of the sediment cores (interior samples) contained generally less PFT than the core margins (exterior samples) and thus have lower potential drilling fluid (DF) contamination. The majority of sediment samples (interior) at Sites U1370 and U1371 have a contamination potential close to or below detection levels (i.e., $1.19 \times 10^{-4}$ $\mathrm{ng}_{\mathrm{PFT}} / \mathrm{g}_{\text {sediment }}$ or $1.78 \times 10^{-3} \mu \mathrm{L}_{\mathrm{DF}} / \mathrm{g}_{\text {sediment }}$ on average $)$. We observed higher contamination values (i.e., $7.28 \times 10^{-3} \mathrm{ng} \mathrm{PFT} / \mathrm{g}_{\text {sediment }}$ or $6.98 \times 10^{-2} \mu \mathrm{L}_{\mathrm{DF}} / \mathrm{g}_{\text {sediment }}$ on average) at Sites U1365, U1366, and U1367. Finally, we measured much higher PFT concentrations throughout the sediment of Sites U1368 and U1369 (i.e., $5.48 \times$ $10^{-2} \mathrm{ng}_{\text {PFT }} / \mathrm{g}_{\text {sediment }}$ or $6.60 \times 10^{-1} \mu \mathrm{L}_{\mathrm{DF}} / \mathrm{g}_{\text {sediment }}$ on average). We observe no apparent correlation of sample PFT content to sediment lithology, degree of sediment disturbance, core section number, or porosity.

\section{Introduction}

Integrated Ocean Drilling Program (IODP) Expedition 329 to the South Pacific Gyre was conducted to document the extent and character of life in sedimentary habitats with very low biomass and rates of activity (D'Hondt et al., 2013). To meet these objectives, Expedition 329 scientists cored the entire sediment column at seven sites along two transects in the South Pacific Gyre (see the "Expedition 329 summary" chapter [Expedition 329 Scientists, 2011]). Characterization of microbial communities in the recovered sediment requires precise constraints on the maximum potential for microbiological contamination associated with the 
coring process. Surface seawater used as the drilling fluid is a ubiquitous potential contaminating medium (Smith et al., 2000). With about three orders of magnitude difference between in situ (sediment) cell abundances and cell abundances in South Pacific Gyre surface seawater, the potential for microbial contamination is of major concern (see $\mathrm{D}^{\prime}$ Hondt et al., 2011; D'Hondt et al., 2015). We monitored the intrusion potential of drilling fluid during the coring operations by adding a water-soluble perfluorocarbon tracer (PFT) to the drilling fluid and subsequently measuring the PFT concentration in sediment samples immediately adjacent to dedicated microbiology samples. The addition of PFT to drilling fluid is an effective way to quantify drilling-induced contamination, as it is inert and can be detected with high sensitivity (Colwell et al., 1992). The measured PFT concentration in the sediment can then be used as an indirect measure of drilling fluid intrusion and potential associated entrainment of nonindigenous cells. PFT analyses have been successfully applied to detect contaminants prior to microbiological studies on numerous other deep-biosphere projects, such as Ocean Drilling Program (ODP) Leg 201 (House et al., 2003) and IODP Expedition 301 (Lever et al., 2006).

The R/V JOIDES Resolution piston cored sediment at all seven sites (IODP Sites U1365-U1371) during Expedition 329 (Fig. F1; see the "Expedition 329 summary" chapter [Expedition 329 Scientists, 2011]). A total of 556 sediment samples were recovered from these sites to analyze their PFT content for (1) quantification of potential drilling fluid intrusion and (2) introduction of nonindigenous cells during coring. The primary objective of these analyses was to identify core intervals with the least potential coring contamination for microbiological studies.

\section{Methods}

\section{PFT delivery}

PFT was introduced into the drilling fluid (DF) stream at a rate calculated to achieve a concentration of $0.89 \mathrm{mg}_{\mathrm{PFT}} / \mathrm{L}_{\mathrm{DF}}$. The injection rate was controlled with a single-piston high-pressure liquid chromatography (HPLC) pump, which pumps the PFT from a polypropylene carboy into the drilling mud stream. For each hole, we started pumping the tracer early enough to ensure that the tracer reached the drill bit well before drilling operations began. We kept track of drilling fluid PFT concentrations by monitoring the drilling mud pump rate (in liters per minute) and the PFT delivery rate (in milliliters PFT per minute) using shipboard instrumentation software (an example for Site U1370 is shown in Fig. F2).

\section{Sample collection}

Unconsolidated sediment was sampled on the catwalk for PFT analyses immediately after core retrieval. PFT samples were consistently sampled adjacent to the section/whole round that had been selected for microbiological analysis. Two cylindrical plugs $\left(3 \mathrm{~cm}^{3}\right.$ each) were taken from the bottom end of each section with $5 \mathrm{~mL}$ cut-off syringes. One plug was taken from the outer edge near the core liner to confirm successful delivery of the tracer (exterior samples) and the other was taken from near the center of the core to quantify drill water intrusion to the core center (interior samples). The samples were immediately extruded into $20 \mathrm{~mL}$ capacity headspace glass vials containing iso-octane (Sites U1365 and U1366) or water (Sites U1367-U1371) and sealed with gas-tight caps containing septa.

\section{PFT analysis (postcruise)}

The low solubility of PFT in water facilitates gas partitioning, allowing a quantitative headspace analysis via gas chromatography (GC). Due to the lack of an appropriate GC column, PFT measurements were not possible during the expedition. Therefore, we quantified the PFT concentration in the samples postcruise using a Shimadzu 17A gas chromatograph with an electron capture detector (ECD) at the Graduate School of Oceanography, University of Rhode Island. We heated the headspace vials containing the sediment samples for at least $30 \mathrm{~min}$ in a $70^{\circ} \mathrm{C}$ oven in order to release the tracer from the sediment. We directly injected $2.0 \mathrm{~mL}$ of headspace gas from samples, standards, and blanks manually using $3 \mathrm{~mL}$ plastic syringes and needles. Syringes and needles were similarly heated for $30 \mathrm{~min}$ at $70^{\circ} \mathrm{C}$ prior to injection in order to minimize absorption of PFT into the interior of the syringe and to negate sample cross-contamination. The oven temperature was set $6^{\circ} \mathrm{C}$ lower than the PFT boiling point $\left(76^{\circ} \mathrm{C}\right)$ to avoid melting the plastic syringes during the heating process. Because the temperature was slightly below the PFT boiling point, PFT extraction may not have been complete. However, because PFT readily evaporates due to its inherent low viscosity and low surface tension, we believe that most of the PFT was extracted. For description of a detailed analytical protocol, we refer to Smith et al. (2000) and House et al. (2003). 


\section{Calibration, instrumental and procedural blanks, and detection limit}

For standards, we diluted PFT in ultrapure methanol to $10^{-2}, 10^{-4}, 10^{-6}$, and $10^{-8} \mathrm{vol}: \mathrm{vol}$ in $20 \mathrm{~mL}$ capacity headspace vials (see Appendix A). We constructed a standard curve by plotting the PFT peak area versus PFT mass injected, resulting in a log-log correlation. We calculated the amount of PFT (in grams) contained within an injection by using the formula described in Appendix A. Further details on GC calibration slopes are presented in Appendix B. We analyzed both procedural and instrumental blanks to determine experimental backgrounds. Each instrumental blank consisted of a $2 \mathrm{~mL}$ injection of air collected in a heated gas-tight syringe from outside the laboratory. We also ran procedural blanks consisting of a $2 \mathrm{~mL}$ injection of ambient coring air (headspace gas sealed in an empty vial at time of sample collection), when available. Procedural blanks were collected for Holes U1367D, U1370E, U1370F, and U1371E. To calculate the detection limit, we used the average value and standard deviation of all blank runs combined. We present further details regarding detection limit analysis in Appendix C.

\section{Estimates of maximum potential of drilling fluid intrusion and nonindigenous cells}

We calculated the volume of drilling water intrusion based on the PFT content of each sample and assuming a PFT concentration of $0.89 \mathrm{mg} / \mathrm{L}$ in the drilling fluid. A detailed description of the calculation method is given in Appendix D. We then used the calculated volume of drilling water in each sample to estimate the potential number of contaminant cells. This estimate is usually based on the measured density of cells in the drilling fluid (Lever et al., 2006). However, because no drill water samples were taken for cell counts during Expedition 329 coring operations, we used the average surface seawater cell count from the 2006 site survey expedition, KNOX02RR $\left(1.7 \times 10^{5}\right.$ cells $/ \mathrm{mL}$; see $\mathrm{D}^{\prime}$ Hondt et al., 2011).

\section{Results}

Table T1 presents the detection limits (in nanograms PFT), drilling fluid equivalents (microliters), and nonindigenous cells equivalents (cells) for each of the Expedition 329 sites. The PFT detection limits reported for Expedition 329 sites were in the range of $1.03 \times 10^{-3}$ to $2.17 \times 10^{-3} \mathrm{ng}$ PFT or $1.16 \times 10^{-3}$ to $2.44 \times 10^{-3} \mu \mathrm{L}$ potential seawater intrusion. Variations in detection limit were driven by variations in the instrument calibration obtained for each site during the course of the analysis. Tables T2, T3, T4, T5, T6, T7, and T8 contain the measured PFT content (interior and exterior sample pairs), derived drilling fluid intrusion, and nonindigenous cell entrainment for all analyzed samples, sorted by site and hole. These tables also report sample location, lithology, and degree of sediment disturbance for each analyzed sample. Samples that produced a peak area below their hole's detection limit are denoted as "BD." Downhole variations in PFT content at Expedition 329 sites are shown in Figures F3, F4, F5, F6, F7, F8, and F9 on a logarithmic scale. In these figures, intervals characterized by slight to severe coring disturbance intensity are annotated with shaded areas.

The centers of the sediment cores contain generally less PFT than the core margins. Detection of the tracer in exterior core samples confirms successful delivery, whereas lower concentrations within the interior samples generally demonstrate lower contamination potential in core interiors. Despite these general patterns, PFT concentrations in interior-exterior sample pairs did not always follow a predictable gradient, with maxima at the core liner and minima in the center of the core. More than 50\% of sample pairs in Holes U1365B and U1369C showed elevated PFT content in the center of the core compared to the core margins. These unexpected reversals of the expected pattern might be artifacts of (1) sample mislabeling, (2) differential PFT loss during relatively long sample storage times (months), or (3) advection of drilling fluid through microfissures (Lever et al., 2006). Lever et al. (2006) found relatively higher contamination potential in fine-grained samples (clay) compared to fine sand sediment, possibly due to preferential cracking of clays and resultant advection of drilling fluid through the core interior.

Different degrees of contamination potential characterize the PFT records of different sites. Sites U1370 and U1371 exhibit minimal contamination potential with PFT measurements in 71\%, 99\%, 99\%, and $100 \%$ of the interior samples below detection for Holes U1370E, U1370F, U1371E, and U1371F, respectively. For above detection samples at Sites U1370 and U1371, the detected PFT concentration is relatively low, averaging $8.86 \times 10^{-4} \mathrm{ng}_{\mathrm{PFT}} / \mathrm{g}_{\text {sediment }}$. PFT concentrations were generally above detection levels at Sites U1365-U1369. Intermediate contamination levels (i.e., $7.28 \times 10^{-3} \mathrm{ng}_{\text {PFT }} / g_{\text {sediment }}$ or $6.98 \times 10^{-2}$ $\mu \mathrm{L}_{\mathrm{DF}} / \mathrm{g}_{\text {sediment }}$ on average) were measured at Sites U1365-U1367. Finally, Sites U1368 and U1369 showed contamination potential averaging $9.87 \times$ $10^{-2}$ and $1.09 \times 10^{-2} \mathrm{ng}_{\mathrm{PFT}} / \mathrm{g}_{\text {sediment, }}$ respectively. A large fraction of the sediment from Hole U1368C was reported as slightly disturbed by the shipboard 
scientific party, which may partly explain the elevated PFT content observed for that hole.

To understand the large variation in measured PFT content throughout Expedition 329 sites, we looked for relationships between observed PFT concentration and other parameters, including core section number, core depth, porosity, sediment lithology, and degree of coring disturbance. We found elevated PFT content in 55\% of the samples from sedimentary intervals characterized by shipboard sedimentologists as heavily disturbed. We observed no relationship between lithology (e.g., abyssal clay, carbonate ooze, and siliceous ooze) and PFT content. However, because PFT extraction may have been incomplete at $70^{\circ} \mathrm{C}$, we cannot fully preclude the possibility of a relationship between contamination potential and lithology.

\section{Acknowledgments}

This research used samples provided by the Integrated Ocean Drilling Program (IODP). All samples were collected during IODP Expedition 329. We thank the Expedition 329 captain, crew, technicians, and science party of the R/V JOIDES Resolution for their assistance during the expedition. We especially thank Steven Midgley for his postcruise help in providing logging data. We thank Dr. Mark Alexander Lever for very helpful comments. Postexpedition analyses were funded by the National Science Foundation (NSF) Division of Ocean Sciences (grant OCE1130735) and the NSF-funded Center for Dark Energy Biosphere Investigations (grant NSF-OCE0939564). This is a contribution to the Deep Carbon Observatory (DCO). It is Center for Dark Energy Biosphere Investigations (C-DEBI) contribution \#335.

\section{References}

Colwell, F.S., Stormberg, G.J., Phelps, T.J., Birnbaum, S.A., McKinley, J., Rawson, S.A., Veverka, C., Goodwin, S., Long, P.E., Russell, B.F., Garland, T., Thompson, D., Skinner, P., and Grover, S., 1992. Innovative techniques for collection of saturated and unsaturated subsurface basalts and sediments for microbiological characterization. Journal of Microbiological Methods, 15(4):279-292. http://dx.doi.org/10.1016/0167-7012(92)90047-8

D'Hondt, S., Abrams, L.J., Anderson, R., Dorrance, J., Durbin, A., Ellett, L., Ferdelman, T., Fischer, J., Forschner, S., Fuldauer, R., Goldstein, H., Graham, D., Griffith, W., Halm, H., Harris, R., Harrison, B., Hasiuk, F., Horn, G., Kallmeyer, J., Lever, M., Meyer, J., Morse, L., Moser, C., Murphy, B., Nordhausen, A., Parry, L., Pockalny, R., Puschell, A., Rogers, J., Schrum, H., Smith,
D.C., Soffientino, B., Spivack, A.J., Stancin, A., Steinman, M., and Walczak, P., 2011. KNOX-02RR: drilling site survey-life in subseafloor sediments of the South Pacific Gyre. In D'Hondt, S., Inagaki, F., Alvarez Zarikian, C.A., and the Expedition 329 Scientists, Proceedings of the Integrated Ocean Drilling Program, 329: Tokyo (Integrated Ocean Drilling Program Management International, Inc.). http://dx.doi.org/10.2204/

iodp.proc.329.112.2011

D'Hondt, S., Inagaki, F., Alvarez Zarikian, C., Abrams, L.J., Dubois, N., Engelhardt, T., Evans, H., Ferdelman, T., Gribsholt, B., Harris, R.N., Hoppie, B.W., Hyun, J.-H., Kallmeyer, J., Kim. J., Lynch, J.E., McKinley, C.C., Mitsunobu, S., Morono, Y., Murray, R.W., Pockalny, R., Sauvage, J., Shimono, T., Shiraishi, F., Smith, D.C., SmithDuque, C.E., Spivack, A.J., Steinsbu, B.O., Suzuki, Y., Szpak, M., Toffin, L., Uramoto, G., Yamaguchi, Y.T., Zhang, G., Zhang, X.-H., and Ziebis, W., 2015. Presence of oxygen and aerobic communities from sea floor to basement in deep-sea sediments. Nature Geoscience, 8(4):299-304. http://dx.doi.org/10.1038/ngeo2387

D'Hondt, S., Inagaki, F., Alvarez Zarikian, C.A., and the IODP Expedition 329 Science Party, 2013. IODP Expedition 329: life and habitability beneath the seafloor of the South Pacific Gyre. Scientific Drilling, 15:4-10. http://dx.doi.org/10.2204/iodp.sd.15.01.2013

Expedition 329 Scientists, 2011. Expedition 329 summary. In $\mathrm{D}^{\prime}$ Hondt, S., Inagaki, F., Alvarez Zarikian, C.A., and the Expedition 329 Scientists, Proceedings of the Integrated Ocean Drilling Program, 329: Tokyo (Integrated Ocean Drilling Program Management International, Inc.).

http://dx.doi.org/10.2204/iodp.proc.329.101.2011

House, C.H., Cragg, B.A., Teske, A., and the Leg 201 Scientific Party, 2003. Drilling contamination tests during ODP Leg 201 using chemical and particulate tracers. In D'Hondt, S.L., Jørgensen, B.B., Miller, D.J., et al., Proceedings of the Ocean Drilling Program, Initial Reports, 201: College Station, TX (Ocean Drilling Program), 1-19. http://dx.doi.org/10.2973/odp.proc.ir.201.102.2003

Lever, M.A., Alperin, M., Engelen, B., Inagaki, F., Nakagawa, S., Steinsbu, B.O., Teske A., and IODP Expedition 301 Scientists, 2006. Trends in basalt and sediment core contamination during IODP Expedition 301. Geomicrobiology Journal, 23(7):517-530. http://dx.doi.org/ 10.1080/01490450600897245

Smith, D.C., Spivack, A.J., Fisk, M.R., Haveman, S.A., Staudigel, H., and the Leg 185 Shipboard Scientific Party, 2000. Methods for quantifying potential microbial contamination during deep ocean coring. Ocean Drilling Program Technical Note, 28. http://dx.doi.org/10.2973/ odp.tn.28.2000

Initial receipt: 2 June 2016

Acceptance: 3 October 2016

Publication: 30 January 2017

MS 329-204 
Figure F1. Map of seafloor bathymetry showing Expedition 329 site locations.

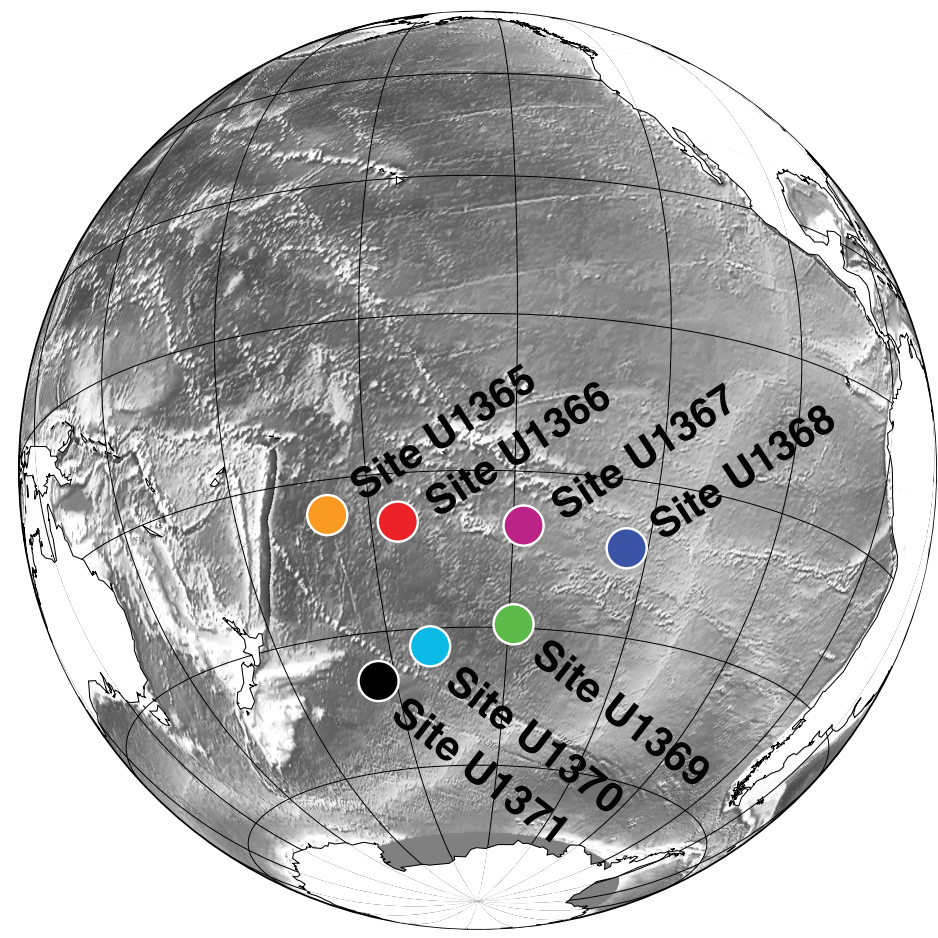

Figure F2. Drilling mud pump rate and perfluorocarbon tracer (PFT) delivery rate monitored during drilling operations and calculated drilling fluid (DF) PFT concentration, Site U1370.
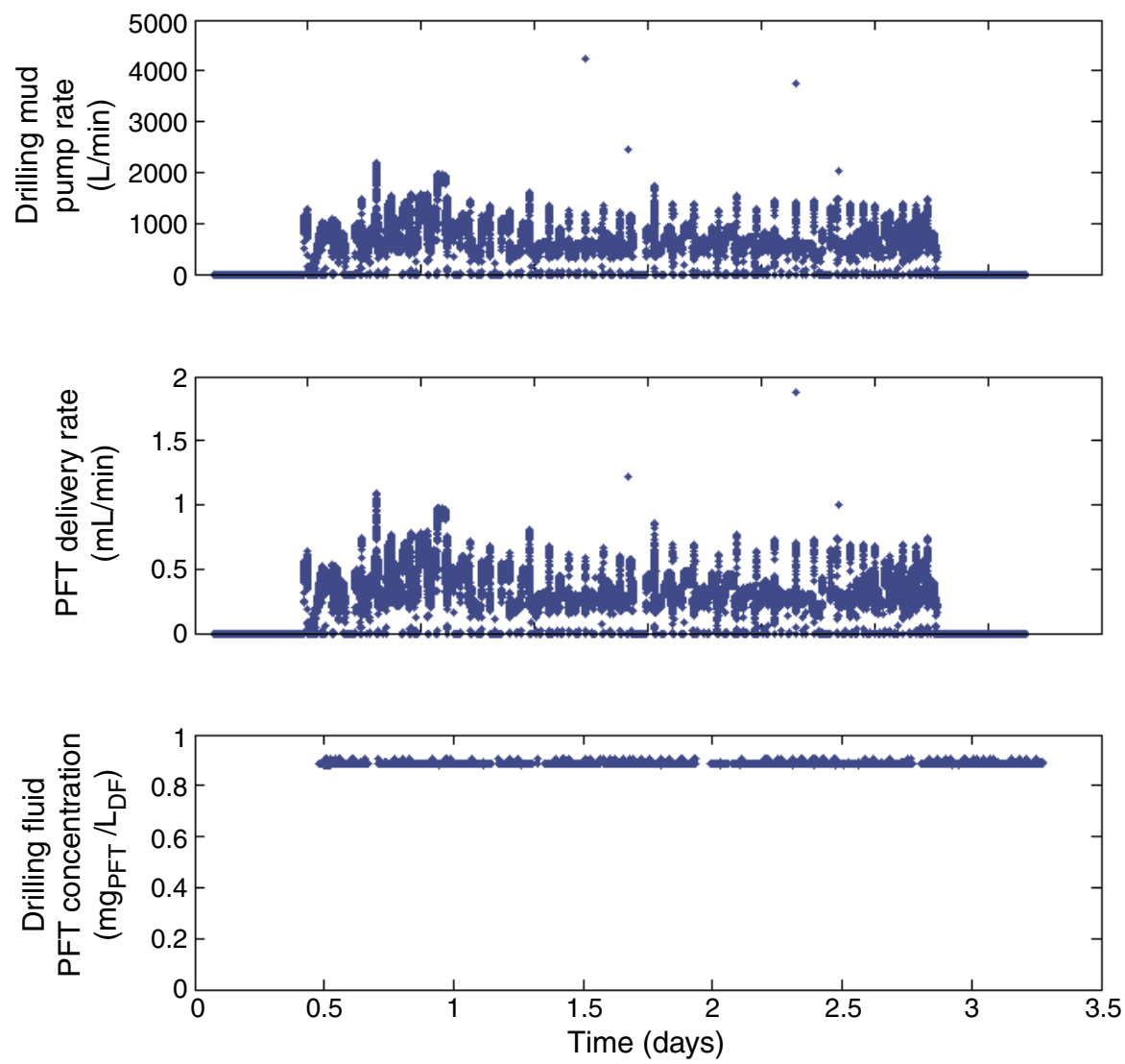
Figure F3. Downcore perfluorocarbon tracer (PFT) concentrations, Site U1365.

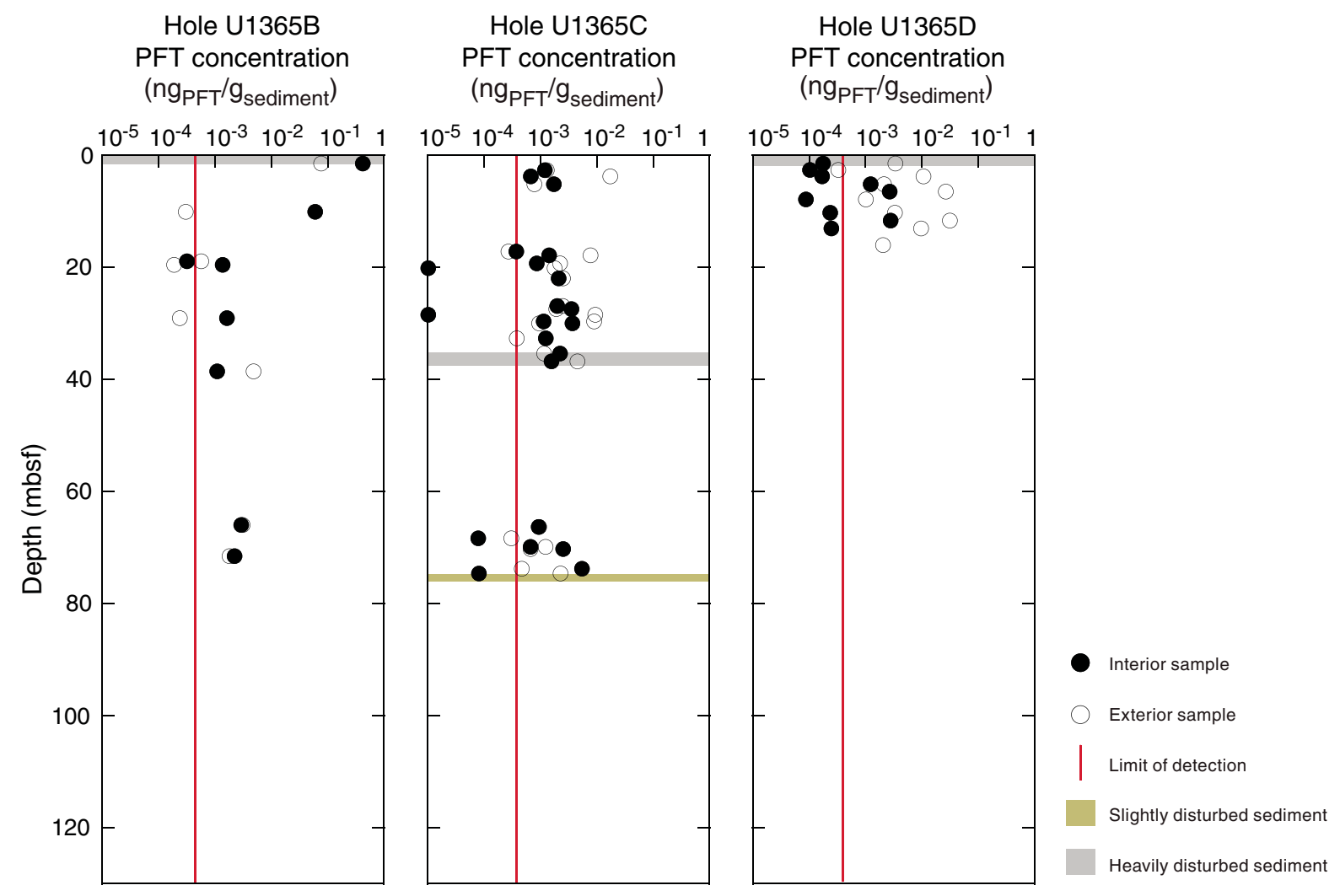

Figure F4. Downcore perfluorocarbon tracer (PFT) concentrations, Site U1366.

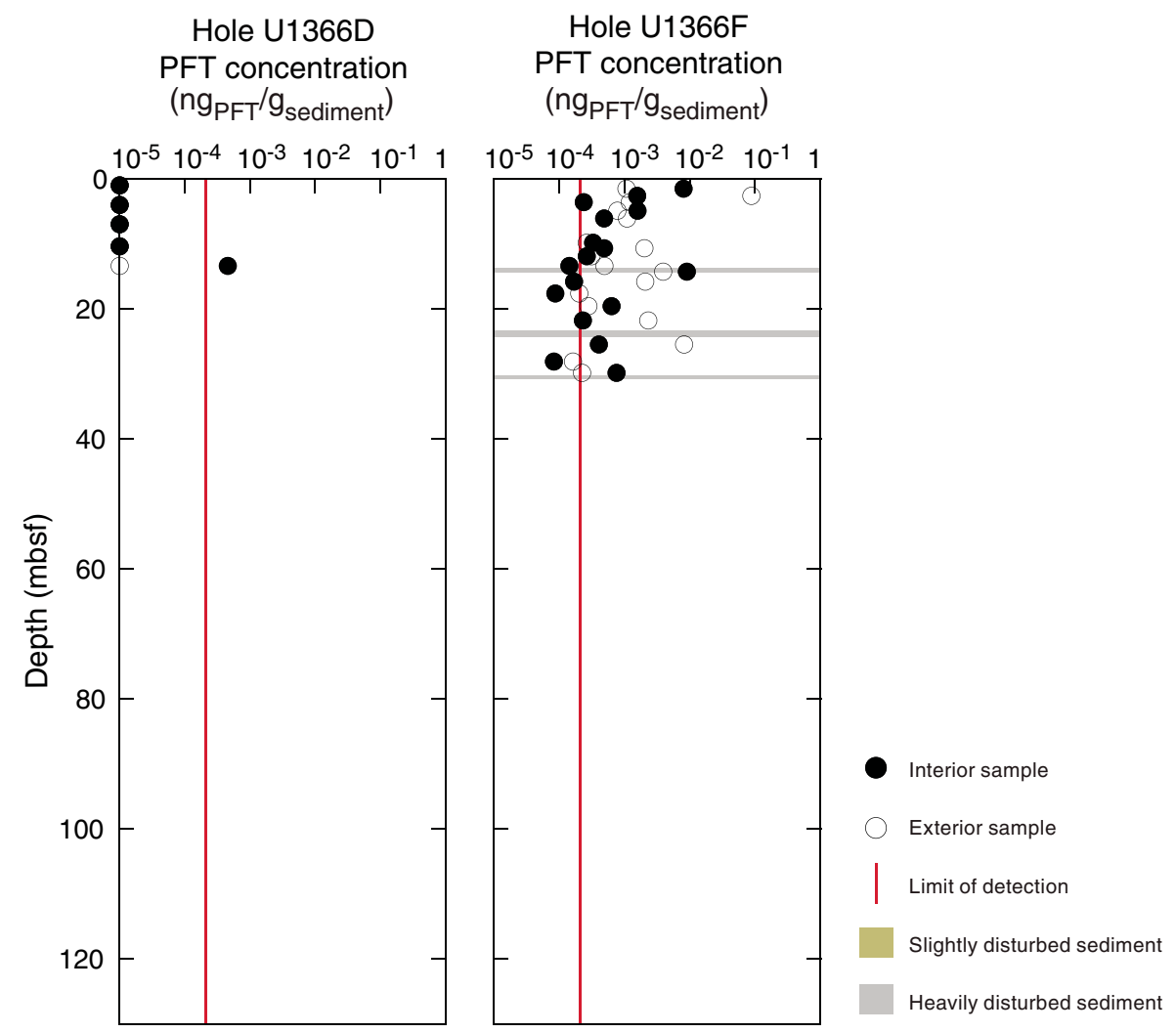


Figure F5. Downcore perfluorocarbon tracer (PFT) concentrations, Site U1367.

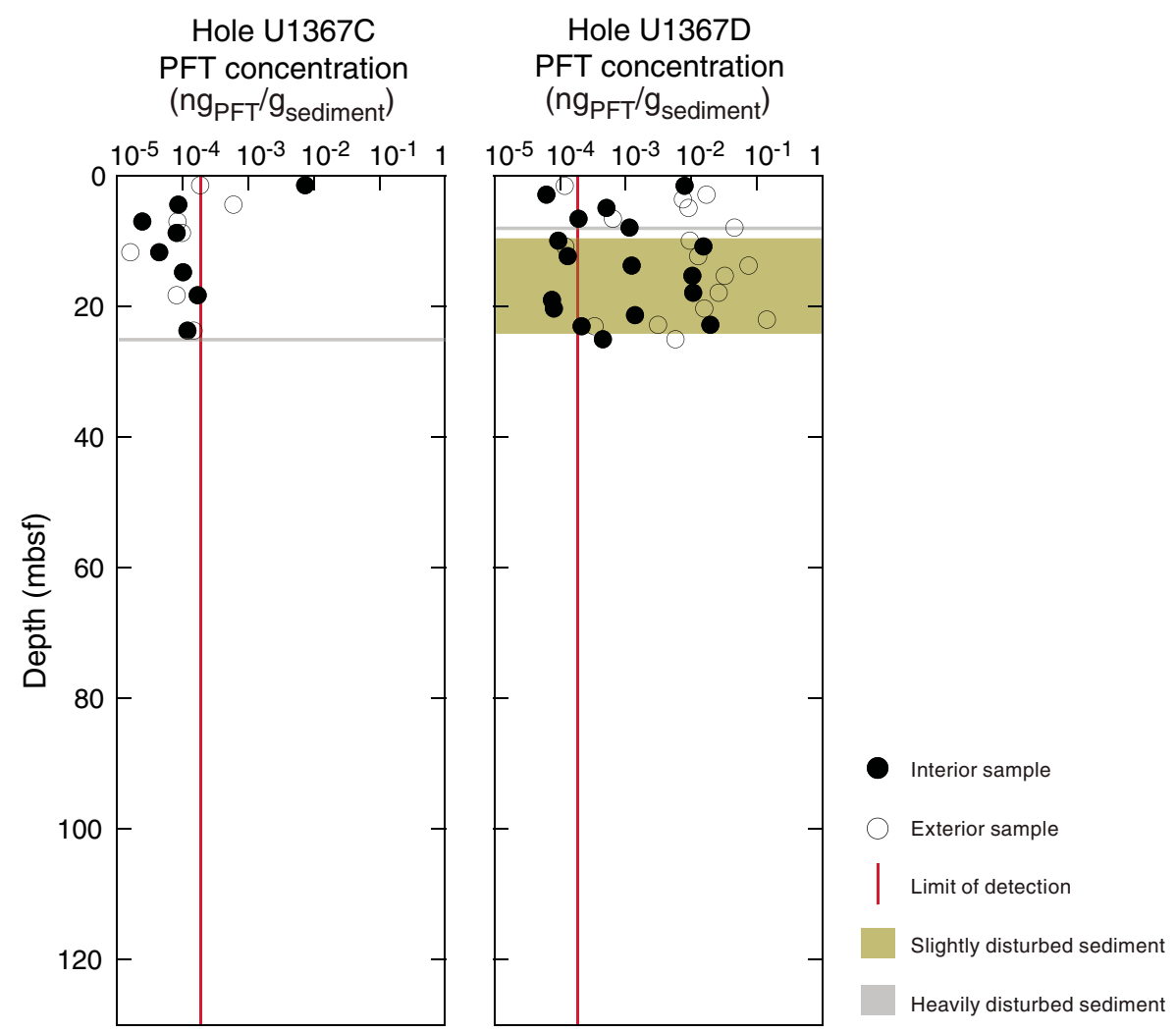

Figure F6. Downcore perfluorocarbon tracer (PFT) concentrations, Site U1368.

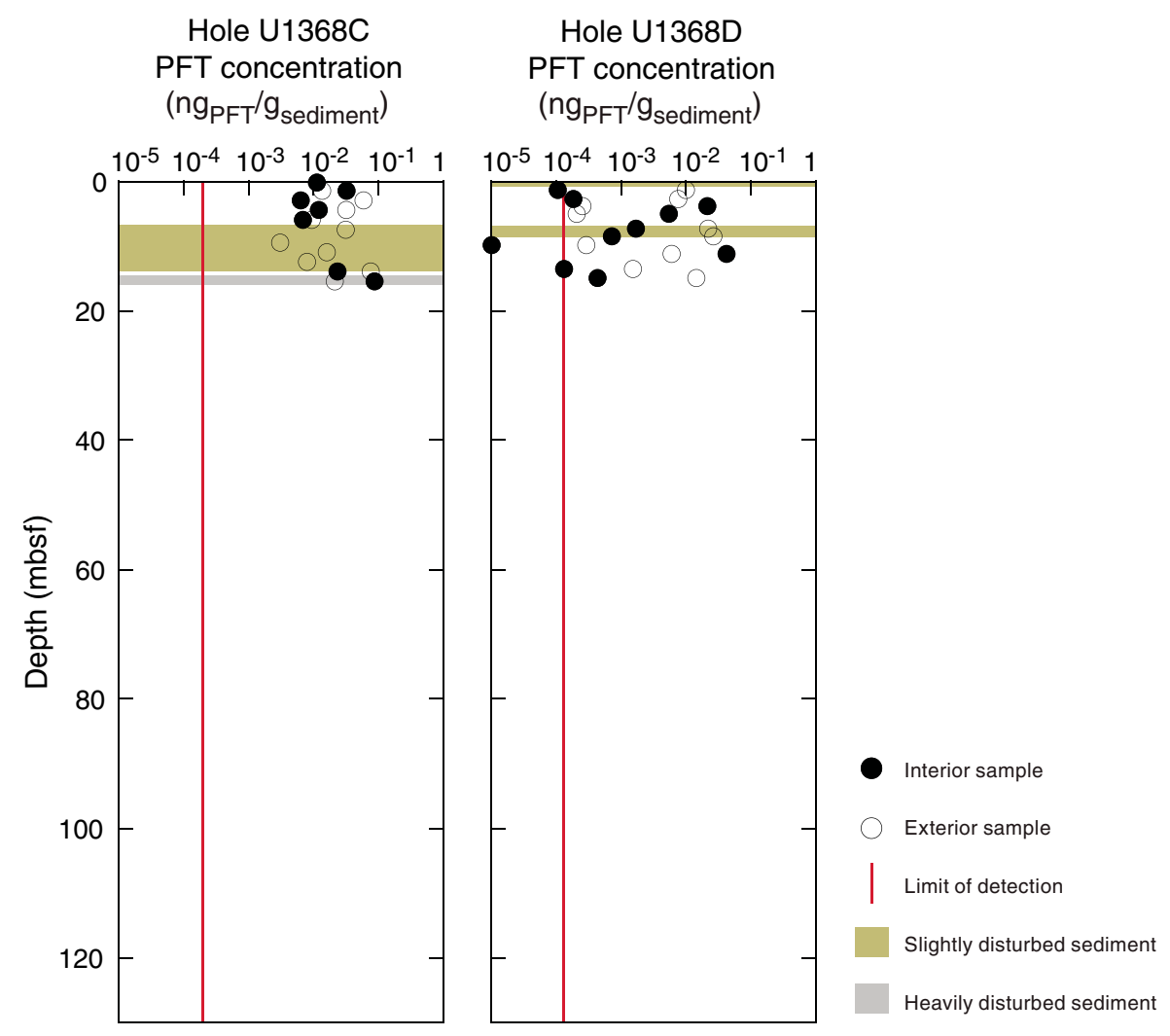


Figure F7. Downcore perfluorocarbon tracer (PFT) concentrations, Site U1369.

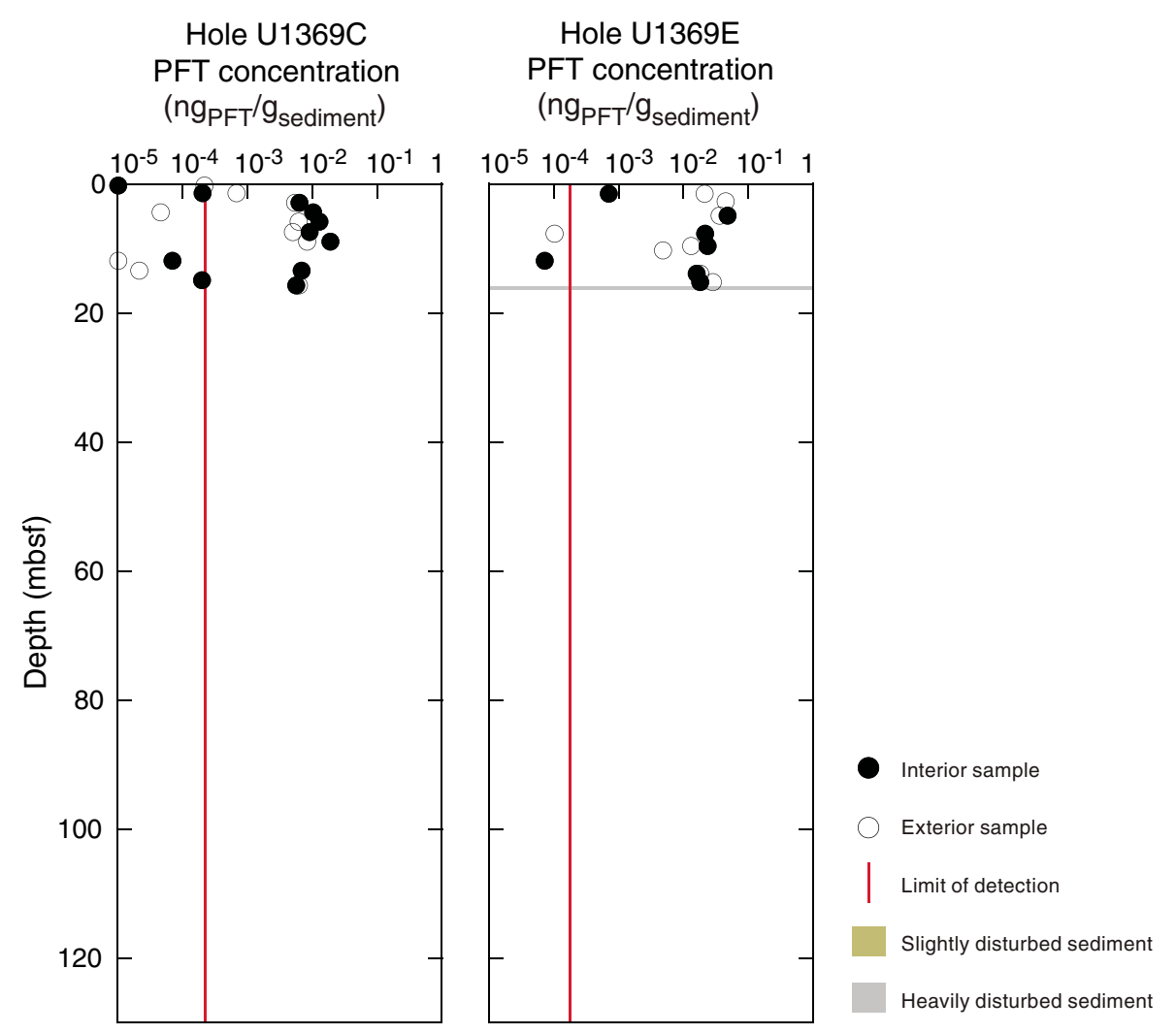

Figure F8. Downcore perfluorocarbon tracer (PFT) concentrations, Site U1370.

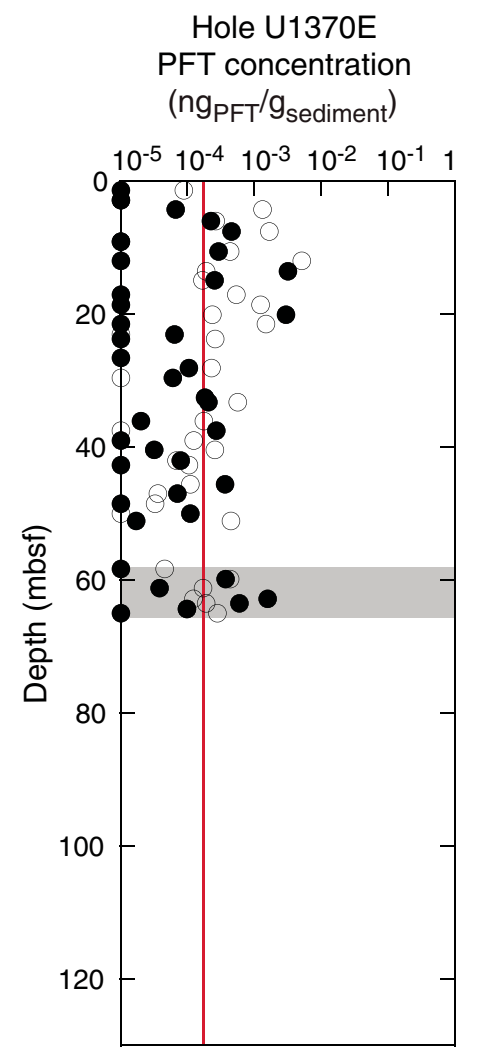

Hole U1370F

PFT concentration

$\left(\mathrm{ng}_{\mathrm{PFT}} / \mathrm{g}_{\text {sediment }}\right)$

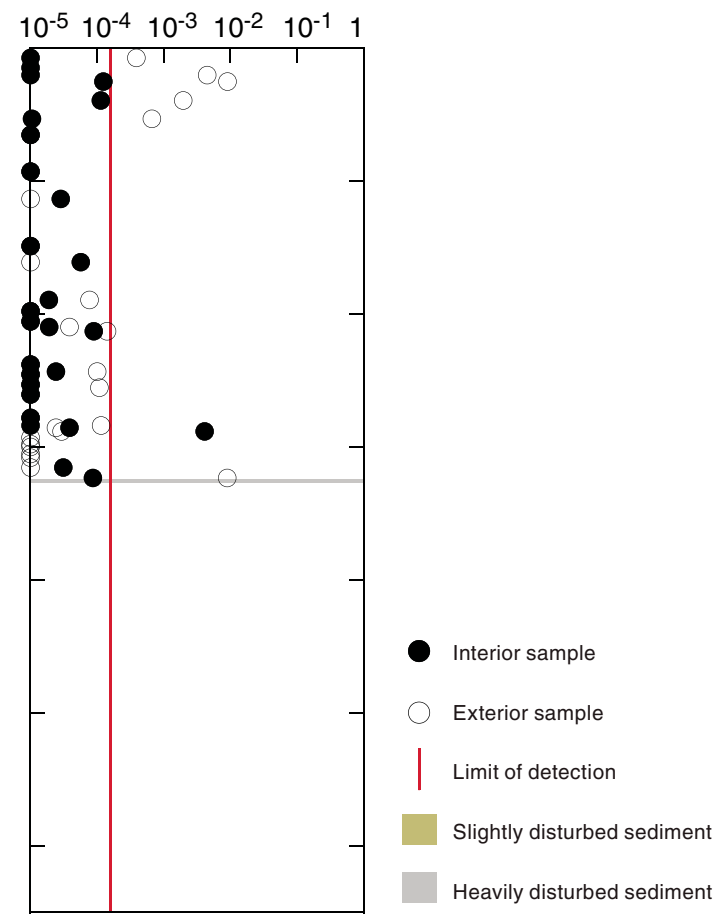


Figure F9. Downcore perfluorocarbon tracer (PFT) concentrations, Site U1371.

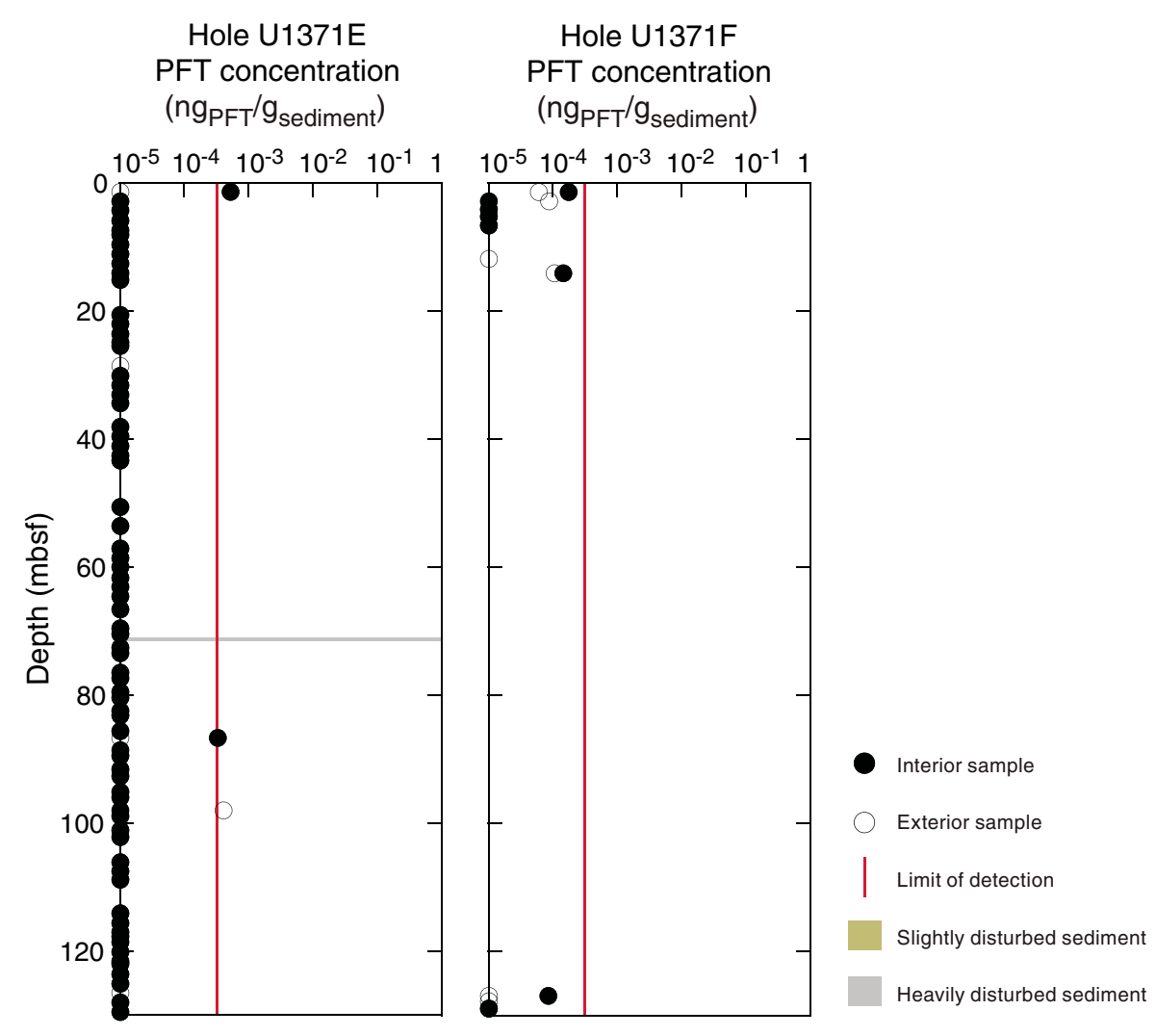


Table T1. Detection limits, drilling fluid equivalents, and nonindigenous cells equivalents, Sites U1365-U1371.

\begin{tabular}{lccc}
\hline & \multicolumn{3}{c}{ Detection limit } \\
\cline { 2 - 4 } Holes & $\begin{array}{c}\text { PFT in sample } \\
\text { (ng) }\end{array}$ & $\begin{array}{c}\text { Drilling fluid } \\
\text { equivalent }(\mu \mathrm{L})\end{array}$ & $\begin{array}{c}\text { Cells equivalent } \\
\text { (cells) }\end{array}$ \\
\hline U1365B-U1365D & $2.17 \mathrm{E}-03$ & $2.44 \mathrm{E}-03$ & 0.4 \\
U1366D and U1366F & $1.06 \mathrm{E}-03$ & $1.19 \mathrm{E}-03$ & 0.2 \\
U1367C and U1367D & $1.18 \mathrm{E}-03$ & $1.32 \mathrm{E}-03$ & 0.2 \\
U1368C and U1368D & $1.03 \mathrm{E}-03$ & $1.16 \mathrm{E}-03$ & 0.2 \\
U1369C and U1369E & $1.29 \mathrm{E}-03$ & $1.46 \mathrm{E}-03$ & 0.2 \\
U1370E and U1370F & $1.33 \mathrm{E}-03$ & $1.50 \mathrm{E}-03$ & 0.3 \\
U1371E and U1371F & $1.65 \mathrm{E}-03$ & $1.86 \mathrm{E}-03$ & 0.3 \\
\hline
\end{tabular}

PFT = perfluorocarbon tracer

Table T2. Perfluorocarbon tracer (PFT) concentration results, Holes U1365B-U1365D.

\begin{tabular}{|c|c|c|c|c|c|c|c|c|c|}
\hline \multirow{3}{*}{$\begin{array}{l}\text { Core, } \\
\text { section }\end{array}$} & \multirow{3}{*}{$\begin{array}{l}\text { Depth } \\
\text { (mbsf) }\end{array}$} & \multirow[b]{3}{*}{ Lithology } & \multirow{3}{*}{$\begin{array}{l}\text { Coring } \\
\text { disturbance } \\
\text { intensity }\end{array}$} & \multicolumn{6}{|c|}{ Contamination } \\
\hline & & & & \multicolumn{3}{|c|}{ Exterior } & \multicolumn{3}{|c|}{ Interior } \\
\hline & & & & $\left(n g_{\mathrm{PFT}} / \mathrm{g}\right)$ & $\left(\mu \mathrm{L}_{\mathrm{DF}} / \mathrm{g}\right)$ & (Cells/g) & $\left(\mathrm{ng}_{\mathrm{PFT}} / \mathrm{g}\right)$ & $\left(\mu \mathrm{L}_{\mathrm{DF}} / \mathrm{g}\right)$ & (Cells/g) \\
\hline \multicolumn{10}{|c|}{ 329-U1365B- } \\
\hline $1 \mathrm{H}-1$ & 1.5 & Metalliferous clay & Very & 7.59E-02 & $5.79 \mathrm{E}-01$ & 99 & $4.15 \mathrm{E}-01$ & $3.73 \mathrm{E}+00$ & 633 \\
\hline $2 \mathrm{H}-4$ & 10.1 & Metalliferous clay & None & BD & BD & BD & $5.92 \mathrm{E}-02$ & $5.74 \mathrm{E}-01$ & 98 \\
\hline $3 \mathrm{H}-4$ & 18.9 & Pelagic clay & None & $\mathrm{BD}$ & $\mathrm{BD}$ & $\mathrm{BD}$ & BD & BD & $\mathrm{BD}$ \\
\hline $3 \mathrm{H}-4$ & 19.6 & Pelagic clay & None & BD & BD & BD & $1.35 \mathrm{E}-03$ & $1.39 \mathrm{E}-02$ & 2 \\
\hline $4 \mathrm{H}-4$ & 29.1 & Pelagic clay & None & BD & $\mathrm{BD}$ & BD & $1.60 \mathrm{E}-03$ & $1.56 \mathrm{E}-02$ & 3 \\
\hline $5 \mathrm{H}-4$ & 38.6 & Zeolitic metalliferous pelagic clay & None & $4.74 \mathrm{E}-03$ & $4.93 \mathrm{E}-02$ & 8 & $1.08 \mathrm{E}-03$ & $1.12 \mathrm{E}-02$ & 2 \\
\hline $8 \mathrm{H}-2$ & 66.0 & Metalliferous clay & None & $3.05 \mathrm{E}-03$ & 2.99E-02 & 5 & $2.89 \mathrm{E}-03$ & $2.94 \mathrm{E}-02$ & 5 \\
\hline $9 \mathrm{H}-3$ & 71.5 & Metalliferous clay & None & $1.78 \mathrm{E}-03$ & $1.72 \mathrm{E}-02$ & 3 & $2.18 \mathrm{E}-03$ & $2.14 \mathrm{E}-02$ & 4 \\
\hline \multicolumn{10}{|c|}{ 329-U1365C- } \\
\hline 1H-2 & 2.7 & Metalliferous clay & None & $1.28 \mathrm{E}-03$ & $1.29 \mathrm{E}-02$ & 2 & 1.17E-03 & $1.20 \mathrm{E}-02$ & 2 \\
\hline $1 \mathrm{H}-3$ & 3.8 & Metalliferous clay & None & $1.71 \mathrm{E}-02$ & $1.78 \mathrm{E}-01$ & 30 & $6.66 \mathrm{E}-04$ & $6.94 \mathrm{E}-03$ & 1 \\
\hline $1 \mathrm{H}-4$ & 5.2 & Metalliferous clay & None & $7.63 \mathrm{E}-04$ & $7.96 \mathrm{E}-03$ & 1 & $1.71 \mathrm{E}-03$ & $1.78 \mathrm{E}-02$ & 3 \\
\hline $3 \mathrm{H}-2$ & 17.2 & Metalliferous clay & None & BD & BD & BD & BD & BD & BD \\
\hline $3 \mathrm{H}-2$ & 17.9 & Metalliferous clay & None & $7.65 \mathrm{E}-03$ & $7.90 \mathrm{E}-02$ & 13 & $1.40 \mathrm{E}-03$ & $1.45 \mathrm{E}-02$ & 2 \\
\hline $3 \mathrm{H}-3$ & 19.3 & Metalliferous clay & None & $2.20 \mathrm{E}-03$ & $2.28 \mathrm{E}-02$ & 4 & $8.49 \mathrm{E}-04$ & $8.94 \mathrm{E}-03$ & 2 \\
\hline $3 \mathrm{H}-4$ & 20.1 & Metalliferous clay & None & $1.76 \mathrm{E}-03$ & $1.83 \mathrm{E}-02$ & 3 & BD & BD & BD \\
\hline $3 \mathrm{H}-5$ & 22.0 & Metalliferous clay & None & $2.47 \mathrm{E}-03$ & $2.54 \mathrm{E}-02$ & 4 & 2.07E-03 & $2.14 \mathrm{E}-02$ & 4 \\
\hline $4 \mathrm{H}-2$ & 26.9 & Metalliferous clay & None & $2.44 \mathrm{E}-03$ & $2.52 \mathrm{E}-02$ & 4 & $1.96 \mathrm{E}-03$ & $2.03 \mathrm{E}-02$ & 3 \\
\hline $4 \mathrm{H}-2$ & 27.5 & Metalliferous clay & None & $1.87 \mathrm{E}-03$ & $1.94 \mathrm{E}-02$ & 3 & $3.51 \mathrm{E}-03$ & $3.70 \mathrm{E}-02$ & 6 \\
\hline $4 \mathrm{H}-3$ & 28.5 & Metalliferous clay & None & $9.31 \mathrm{E}-03$ & $9.65 \mathrm{E}-02$ & 16 & BD & BD & BD \\
\hline $4 \mathrm{H}-4$ & 29.6 & Metalliferous clay & None & $8.79 \mathrm{E}-03$ & $9.07 \mathrm{E}-02$ & 15 & $1.12 \mathrm{E}-03$ & $1.16 \mathrm{E}-02$ & 2 \\
\hline $4 \mathrm{H}-4$ & 30.0 & Metalliferous clay & None & $9.31 \mathrm{E}-04$ & $9.55 \mathrm{E}-03$ & 2 & $3.61 \mathrm{E}-03$ & $3.75 \mathrm{E}-02$ & 6 \\
\hline $4 \mathrm{H}-6$ & 32.7 & Metalliferous clay & None & BD & BD & $\mathrm{BD}$ & $1.23 \mathrm{E}-03$ & $1.28 \mathrm{E}-02$ & 2 \\
\hline $5 \mathrm{H}-1$ & 35.4 & Metalliferous clay & None & $1.15 \mathrm{E}-03$ & $1.24 \mathrm{E}-02$ & 2 & $2.19 \mathrm{E}-03$ & $2.26 \mathrm{E}-02$ & 4 \\
\hline $5 \mathrm{H}-2$ & 36.8 & Metalliferous clay & Very & $4.46 \mathrm{E}-03$ & $4.55 \mathrm{E}-02$ & 8 & $1.55 \mathrm{E}-03$ & $1.58 \mathrm{E}-02$ & 3 \\
\hline $7 \mathrm{H}-3$ & 66.3 & Metalliferous clay & None & $9.35 \mathrm{E}-04$ & $9.27 \mathrm{E}-03$ & 2 & $9.14 \mathrm{E}-04$ & $9.10 \mathrm{E}-03$ & 2 \\
\hline $8 \mathrm{H}-1$ & 68.4 & Chert-metalliferous clay & None & BD & BD & BD & BD & BD & BD \\
\hline $8 \mathrm{H}-2$ & 69.9 & Metalliferous clay & None & $1.21 \mathrm{E}-03$ & $1.19 \mathrm{E}-02$ & 2 & $6.58 \mathrm{E}-04$ & $6.42 \mathrm{E}-03$ & 1 \\
\hline $8 \mathrm{H}-3$ & 70.3 & Metalliferous clay & None & $6.55 \mathrm{E}-04$ & $6.44 \mathrm{E}-03$ & 1 & $2.50 \mathrm{E}-03$ & $2.44 \mathrm{E}-02$ & 4 \\
\hline $8 \mathrm{H}-2$ & 73.7 & Metalliferous clay & None & $4.56 \mathrm{E}-04$ & $4.44 \mathrm{E}-03$ & 1 & $5.35 \mathrm{E}-03$ & $5.10 \mathrm{E}-02$ & 9 \\
\hline $9 \mathrm{H}-3$ & 74.6 & Metalliferous clay & Slightly & $2.24 \mathrm{E}-03$ & $2.25 \mathrm{E}-02$ & 4 & BD & BD & BD \\
\hline \multicolumn{10}{|c|}{ 329-U1365D- } \\
\hline 1H-1 & 1.5 & Metalliferous clay & Very & $3.34 \mathrm{E}-03$ & $3.38 \mathrm{E}-02$ & 6 & $\mathrm{BD}$ & BD & $\mathrm{BD}$ \\
\hline $1 \mathrm{H}-2$ & 2.6 & Metalliferous clay & Slightly & BD & BD & BD & BD & BD & $\mathrm{BD}$ \\
\hline $1 \mathrm{H}-3$ & 3.8 & Metalliferous clay & None & $1.06 \mathrm{E}-02$ & $1.10 \mathrm{E}-01$ & 19 & BD & BD & $\mathrm{BD}$ \\
\hline $1 \mathrm{H}-4$ & 5.2 & Metalliferous clay & None & $2.11 \mathrm{E}-03$ & $2.20 \mathrm{E}-02$ & 4 & $1.22 \mathrm{E}-03$ & $1.29 \mathrm{E}-02$ & 2 \\
\hline $1 \mathrm{H}-5$ & 6.5 & Metalliferous clay & None & $2.66 \mathrm{E}-02$ & $2.76 \mathrm{E}-01$ & 47 & $2.66 \mathrm{E}-03$ & $2.72 \mathrm{E}-02$ & 5 \\
\hline $1 \mathrm{H}-6$ & 7.9 & Metalliferous clay & None & $1.00 \mathrm{E}-03$ & $1.03 \mathrm{E}-02$ & 2 & BD & BD & $\mathrm{BD}$ \\
\hline $2 \mathrm{H}-1$ & 10.3 & Metalliferous clay & None & $3.30 \mathrm{E}-03$ & $3.46 \mathrm{E}-02$ & 6 & $\mathrm{BD}$ & BD & $\mathrm{BD}$ \\
\hline $2 \mathrm{H}-2$ & 11.7 & Metalliferous clay & None & $3.13 \mathrm{E}-02$ & $3.28 \mathrm{E}-01$ & 56 & $2.78 \mathrm{E}-03$ & 2.87E-02 & 5 \\
\hline $2 \mathrm{H}-3$ & 13.1 & Metalliferous clay & None & $9.68 \mathrm{E}-03$ & $1.02 \mathrm{E}-01$ & 17 & BD & BD & BD \\
\hline $2 \mathrm{H}-5$ & 16.0 & Metalliferous clay & None & $2.03 \mathrm{E}-03$ & $2.09 \mathrm{E}-02$ & 4 & NA & NA & NA \\
\hline
\end{tabular}

$\mathrm{BD}=$ below detection, $\mathrm{NA}=$ not applicable. $\mathrm{DF}=$ drilling fluid. 
Table T3. Perfluorocarbon tracer (PFT) concentration results, Holes U1366D and U1366F.

\begin{tabular}{|c|c|c|c|c|c|c|c|c|c|}
\hline \multirow{3}{*}{$\begin{array}{l}\text { Core, } \\
\text { section }\end{array}$} & \multirow{3}{*}{$\begin{array}{l}\text { Depth } \\
\text { (mbsf) }\end{array}$} & \multirow[b]{3}{*}{ Lithology } & \multirow{3}{*}{$\begin{array}{l}\text { Coring } \\
\text { disturbance } \\
\text { intensity }\end{array}$} & \multicolumn{6}{|c|}{ Contamination } \\
\hline & & & & \multicolumn{3}{|c|}{ Exterior } & \multicolumn{3}{|c|}{ Interior } \\
\hline & & & & $\left(\mathrm{ng}_{\mathrm{PFT}} / \mathrm{g}\right)$ & $\left(\mu \mathrm{L}_{\mathrm{DF}} / \mathrm{g}\right)$ & (Cells/g) & $\left(n g_{\mathrm{PFT}} / \mathrm{g}\right)$ & $\left(\mu \mathrm{L}_{\mathrm{DF}} / \mathrm{g}\right)$ & (Cells/g) \\
\hline \multicolumn{10}{|c|}{ 329-U1366D- } \\
\hline $1 \mathrm{H}-1$ & 1.0 & Metalliferous clay & Slightly & BD & BD & BD & $\mathrm{BD}$ & $\mathrm{BD}$ & $\mathrm{BD}$ \\
\hline $1 \mathrm{H}-3$ & 4.0 & Metalliferous clay & None & $\mathrm{BD}$ & $\mathrm{BD}$ & $\mathrm{BD}$ & $\mathrm{BD}$ & $\mathrm{BD}$ & $\mathrm{BD}$ \\
\hline $1 \mathrm{H}-5$ & 7.0 & Metalliferous clay & None & $\mathrm{BD}$ & $\mathrm{BD}$ & $\mathrm{BD}$ & $\mathrm{BD}$ & $\mathrm{BD}$ & $\mathrm{BD}$ \\
\hline $2 \mathrm{H}-1$ & 10.4 & Metalliferous clay & None & BD & BD & BD & $\mathrm{BD}$ & BD & $\mathrm{BD}$ \\
\hline $2 \mathrm{H}-3$ & 13.4 & Metalliferous clay & None & BD & BD & BD & $4.55 \mathrm{E}-04$ & $4.60 \mathrm{E}-03$ & 1 \\
\hline \multicolumn{10}{|c|}{ 329-U1366F- } \\
\hline $1 \mathrm{H}-1$ & 1.5 & Metalliferous clay & None & $1.06 \mathrm{E}-03$ & $1.04 \mathrm{E}-02$ & 2 & $7.88 \mathrm{E}-03$ & $7.88 \mathrm{E}-02$ & 13 \\
\hline $1 \mathrm{H}-2$ & 2.6 & Metalliferous clay & None & $8.68 \mathrm{E}-02$ & $8.60 \mathrm{E}-01$ & 146 & $1.55 \mathrm{E}-03$ & $1.52 \mathrm{E}-02$ & 3 \\
\hline $1 \mathrm{H}-3$ & 3.6 & Metalliferous clay & None & $1.19 \mathrm{E}-03$ & $1.16 \mathrm{E}-02$ & 2 & $2.35 \mathrm{E}-04$ & $2.27 \mathrm{E}-03$ & 0 \\
\hline $1 \mathrm{H}-4$ & 4.9 & Metalliferous clay & None & $7.64 \mathrm{E}-04$ & $7.59 \mathrm{E}-03$ & 1 & $1.57 \mathrm{E}-03$ & $1.57 \mathrm{E}-02$ & 3 \\
\hline $2 \mathrm{H}-1$ & 6.1 & Metalliferous clay & None & $1.08 \mathrm{E}-03$ & $1.06 \mathrm{E}-02$ & 2 & $4.85 \mathrm{E}-04$ & $4.74 \mathrm{E}-03$ & 1 \\
\hline $2 \mathrm{H}-3$ & 9.8 & Metalliferous clay & None & $2.62 \mathrm{E}-04$ & $2.61 \mathrm{E}-03$ & 0 & $3.27 \mathrm{E}-04$ & $3.26 \mathrm{E}-03$ & 1 \\
\hline $2 \mathrm{H}-4$ & 10.7 & Metalliferous clay & None & $1.99 \mathrm{E}-03$ & 1.99E-02 & 3 & $4.84 \mathrm{E}-04$ & $4.74 \mathrm{E}-03$ & 1 \\
\hline $2 \mathrm{H}-5$ & 11.9 & Metalliferous clay & None & $3.08 \mathrm{E}-04$ & $3.00 \mathrm{E}-03$ & 1 & $2.62 \mathrm{E}-04$ & $2.56 \mathrm{E}-03$ & 0 \\
\hline $2 \mathrm{H}-6$ & 13.4 & Metalliferous clay & None & $4.86 \mathrm{E}-04$ & $4.81 \mathrm{E}-03$ & 1 & BD & BD & BD \\
\hline $3 \mathrm{H}-1$ & 14.3 & Metalliferous clay & Very & $3.85 \mathrm{E}-03$ & $3.79 \mathrm{E}-02$ & 6 & $8.91 \mathrm{E}-03$ & $8.79 \mathrm{E}-02$ & 15 \\
\hline $3 \mathrm{H}-2$ & 15.8 & Metalliferous clay & None & $2.06 \mathrm{E}-03$ & $2.06 \mathrm{E}-02$ & 4 & $1.66 \mathrm{E}-04$ & $1.65 \mathrm{E}-03$ & 0 \\
\hline $3 \mathrm{H}-3$ & 17.6 & Metalliferous clay & None & BD & BD & BD & BD & BD & BD \\
\hline $3 \mathrm{H}-4$ & 19.6 & Metalliferous clay & None & $2.76 \mathrm{E}-04$ & $2.78 \mathrm{E}-03$ & 0 & $6.27 \mathrm{E}-04$ & $6.28 \mathrm{E}-03$ & 1 \\
\hline $3 \mathrm{H}-6$ & 21.8 & Metalliferous clay & None & $2.28 \mathrm{E}-03$ & $2.25 \mathrm{E}-02$ & 4 & BD & BD & BD \\
\hline $4 \mathrm{H}-2$ & 25.5 & Metalliferous clay & Very & $8.05 \mathrm{E}-03$ & $8.04 \mathrm{E}-02$ & 14 & $4.02 \mathrm{E}-04$ & $4.11 \mathrm{E}-03$ & 1 \\
\hline $4 \mathrm{H}-4$ & 28.1 & Metalliferous clay & None & BD & BD & $\mathrm{BD}$ & BD & BD & $\mathrm{BD}$ \\
\hline $4 \mathrm{H}-5$ & 29.8 & Metalliferous clay & Very & $2.20 \mathrm{E}-04$ & $2.12 \mathrm{E}-03$ & 0 & 7.47E-04 & $7.48 \mathrm{E}-03$ & 1 \\
\hline
\end{tabular}

$\mathrm{BD}=$ below detection $. \mathrm{DF}=$ drilling fluid.

Table T4. Perfluorocarbon tracer (PFT) concentration results, Holes U1367C and U1367D.

\begin{tabular}{|c|c|c|c|c|c|c|c|c|c|}
\hline \multirow{3}{*}{$\begin{array}{c}\text { Core, } \\
\text { section }\end{array}$} & \multirow{3}{*}{$\begin{array}{l}\text { Depth } \\
\text { (mbsf) }\end{array}$} & \multirow[b]{3}{*}{ Lithology } & \multirow{3}{*}{$\begin{array}{c}\text { Coring } \\
\text { disturbance } \\
\text { intensity }\end{array}$} & \multicolumn{6}{|c|}{ Contamination } \\
\hline & & & & \multicolumn{3}{|c|}{ Exterior } & \multicolumn{3}{|c|}{ Interior } \\
\hline & & & & $\left(\mathrm{ng}_{\mathrm{PFT}} / \mathrm{g}\right)$ & $\left(\mu \mathrm{L}_{D F} / \mathrm{g}\right)$ & (Cells/g) & $\left(\mathrm{ng}_{\mathrm{PFT}} / \mathrm{g}\right)$ & $\left(\mu \mathrm{L}_{D F} / \mathrm{g}\right)$ & (Cells/g) \\
\hline \multicolumn{10}{|c|}{ 329-U1367C- } \\
\hline 1H-1 & 1.4 & Metalliferous clay & None & $1.82 \mathrm{E}-04$ & $2.16 \mathrm{E}-03$ & 0 & $7.26 \mathrm{E}-03$ & 8.23E-02 & 14 \\
\hline $1 \mathrm{H}-3$ & 4.4 & Metalliferous clay & None & $5.81 \mathrm{E}-04$ & 7.06E-03 & 1 & BD & BD & $\mathrm{BD}$ \\
\hline $1 \mathrm{H}-5$ & 6.9 & Nannofossil ooze & Slightly & BD & BD & BD & BD & BD & BD \\
\hline $2 \mathrm{H}-1$ & 8.7 & Nannofossil ooze & None & BD & BD & BD & BD & BD & BD \\
\hline $2 \mathrm{H}-3$ & 11.7 & Nannofossil ooze & None & $\mathrm{BD}$ & BD & BD & BD & BD & BD \\
\hline $2 \mathrm{H}-5$ & 14.7 & Clay-bearing nannofossil ooze & None & NA & NA & NA & $\mathrm{BD}$ & BD & BD \\
\hline $3 \mathrm{H}-2$ & 18.2 & Clay-bearing nannofossil ooze & None & $\mathrm{BD}$ & BD & BD & $\mathrm{BD}$ & BD & BD \\
\hline $3 \mathrm{H}-6$ & 23.6 & Nannofossil chalk & Flow in; slurry & BD & BD & BD & BD & BD & BD \\
\hline \multicolumn{10}{|c|}{ 329-U1367D- } \\
\hline $1 \mathrm{H}-1$ & 1.5 & Metalliferous clay & None & BD & BD & BD & $7.84 \mathrm{E}-03$ & $1.02 \mathrm{E}-01$ & 17 \\
\hline $1 \mathrm{H}-2$ & 2.9 & Metalliferous clay & None & $1.69 \mathrm{E}-02$ & $3.02 \mathrm{E}-01$ & 51 & BD & BD & BD \\
\hline $1 \mathrm{H}-3$ & 3.6 & Metalliferous clay & None & $7.42 \mathrm{E}-03$ & $1.06 \mathrm{E}-01$ & 18 & $5.08 \mathrm{E}-04$ & $6.60 \mathrm{E}-03$ & 1 \\
\hline $1 \mathrm{H}-4$ & 4.9 & Metalliferous clay & None & $9.02 \mathrm{E}-03$ & $1.18 \mathrm{E}-01$ & 20 & NA & NA & NA \\
\hline $1 \mathrm{H}-5$ & 6.5 & Metalliferous clay & None & $6.30 \mathrm{E}-04$ & $8.18 \mathrm{E}-03$ & 1 & BD & BD & BD \\
\hline $2 \mathrm{H}-1$ & 7.9 & Nannofossil ooze & Very & $4.50 \mathrm{E}-02$ & $5.28 \mathrm{E}-01$ & 90 & $1.14 \mathrm{E}-03$ & $1.34 \mathrm{E}-02$ & 2 \\
\hline $2 \mathrm{H}-2$ & 9.9 & Nannofossil ooze & Slightly & $9.46 \mathrm{E}-03$ & $1.25 \mathrm{E}-01$ & 21 & BD & BD & BD \\
\hline $2 \mathrm{H}-3$ & 10.8 & Nannofossil ooze & Slightly & BD & BD & $\mathrm{BD}$ & $1.52 \mathrm{E}-02$ & $1.90 \mathrm{E}-01$ & 32 \\
\hline $2 \mathrm{H}-4$ & 12.3 & Nannofossil ooze & Slightly & $1.27 \mathrm{E}-02$ & $1.55 \mathrm{E}-01$ & 26 & BD & BD & $\mathrm{BD}$ \\
\hline $2 \mathrm{H}-5$ & 13.7 & Nannofossil ooze & Slightly & $7.41 \mathrm{E}-02$ & $8.69 \mathrm{E}-01$ & 148 & $1.22 \mathrm{E}-03$ & $1.45 \mathrm{E}-02$ & 2 \\
\hline $2 \mathrm{H}-6$ & 15.3 & Clay-bearing nannofossil ooze & Slightly & $3.19 \mathrm{E}-02$ & $4.25 \mathrm{E}-01$ & 72 & $1.03 \mathrm{E}-02$ & $1.26 \mathrm{E}-01$ & 21 \\
\hline $3 \mathrm{H}-1$ & 17.9 & Clay-bearing nannofossil ooze & Slightly & $2.59 \mathrm{E}-02$ & $2.82 \mathrm{E}-01$ & 48 & $1.06 \mathrm{E}-02$ & $1.29 \mathrm{E}-01$ & 22 \\
\hline $3 \mathrm{H}-2$ & 19.0 & Clay-bearing nannofossil ooze & Slightly & 1.57E-02 & $1.95 \mathrm{E}-01$ & 33 & BD & BD & $\mathrm{BD}$ \\
\hline $3 \mathrm{H}-3$ & 20.3 & Clay-bearing nannofossil ooze & Slightly & BD & BD & $\mathrm{BD}$ & 7.95E-05 & $9.59 \mathrm{E}-04$ & 0 \\
\hline $3 \mathrm{H}-3$ & 21.3 & Clay-bearing nannofossil ooze & None & $1.41 \mathrm{E}-01$ & $1.87 \mathrm{E}+00$ & 317 & $1.38 \mathrm{E}-03$ & $1.56 \mathrm{E}-02$ & 3 \\
\hline $3 \mathrm{H}-4$ & 22.8 & Clay-bearing nannofossil ooze & Slightly & $3.08 \mathrm{E}-03$ & $3.65 \mathrm{E}-02$ & 6 & $1.94 \mathrm{E}-02$ & $2.41 \mathrm{E}-01$ & 41 \\
\hline $3 \mathrm{H}-5$ & 23.0 & Clay-bearing nannofossil ooze & None & $3.32 \mathrm{E}-04$ & $3.87 \mathrm{E}-03$ & 1 & BD & BD & $\mathrm{BD}$ \\
\hline $3 \mathrm{H}-6$ & 25.0 & Metalliferous clay & None & $5.69 \mathrm{E}-03$ & $7.20 \mathrm{E}-02$ & 12 & 4.45E-04 & $5.96 \mathrm{E}-03$ & 1 \\
\hline
\end{tabular}

$\mathrm{BD}=$ below detection, $\mathrm{NA}=$ not applicable. $\mathrm{DF}=$ drilling fluid. 
Table T5. Perfluorocarbon tracer (PFT) concentration results, Holes U1368C and U1368D.

\begin{tabular}{|c|c|c|c|c|c|c|c|c|c|}
\hline \multirow{3}{*}{$\begin{array}{l}\text { Core, } \\
\text { section }\end{array}$} & \multirow{3}{*}{$\begin{array}{l}\text { Depth } \\
\text { (mbsf) }\end{array}$} & \multirow[b]{3}{*}{ Lithology } & \multirow{3}{*}{$\begin{array}{l}\text { Coring } \\
\text { disturbance } \\
\text { intensity }\end{array}$} & \multicolumn{6}{|c|}{ Contamination } \\
\hline & & & & \multicolumn{3}{|c|}{ Exterior } & \multicolumn{3}{|c|}{ Interior } \\
\hline & & & & $\left(n g_{\mathrm{PFT}} / \mathrm{g}\right)$ & $\left(\mu \mathrm{L}_{\mathrm{DF}} / \mathrm{g}\right)$ & (Cells/g) & $\left(n g_{\mathrm{PFT}} / \mathrm{g}\right)$ & $\left(\mu \mathrm{L}_{\mathrm{DF}} / \mathrm{g}\right)$ & (Cells/g) \\
\hline \multicolumn{10}{|c|}{ 329-U1368C- } \\
\hline $1 \mathrm{H}-1$ & 0.1 & Clay-bearing nannofossil ooze & None & $2.00 \mathrm{E}+00$ & $2.50 \mathrm{E}+01$ & 4246 & $1.11 \mathrm{E}-02$ & 1.39E-01 & 24 \\
\hline $1 \mathrm{H}-1$ & 1.4 & Clay-bearing nannofossil ooze & None & $1.34 \mathrm{E}-02$ & $1.76 \mathrm{E}-01$ & 30 & $3.18 \mathrm{E}-02$ & $4.52 \mathrm{E}-01$ & 77 \\
\hline $1 \mathrm{H}-2$ & 2.9 & Clay-bearing nannofossil ooze & None & $5.83 \mathrm{E}-02$ & 7.34E-01 & 125 & $6.29 \mathrm{E}-03$ & 7.53E-02 & 13 \\
\hline $1 \mathrm{H}-3$ & 4.4 & Clay-bearing nannofossil ooze & None & $3.14 \mathrm{E}-02$ & $3.80 \mathrm{E}-01$ & 65 & 1.19E-02 & $1.45 \mathrm{E}-01$ & 25 \\
\hline $1 \mathrm{H}-4$ & 5.9 & Clay-bearing nannofossil ooze & None & $9.22 \mathrm{E}-03$ & $1.08 \mathrm{E}-01$ & 18 & $6.80 \mathrm{E}-03$ & 7.81E-02 & 13 \\
\hline $1 \mathrm{H}-5$ & 7.4 & Clay-bearing nannofossil ooze & Slightly & $3.10 \mathrm{E}-02$ & $3.77 \mathrm{E}-01$ & 64 & NA & NA & NA \\
\hline $2 \mathrm{H}-1$ & 9.4 & Clay-bearing nannofossil ooze & Slightly & $3.03 \mathrm{E}-03$ & $3.56 \mathrm{E}-02$ & 6 & $1.53 \mathrm{E}+00$ & $1.77 \mathrm{E}+01$ & 3011 \\
\hline $2 \mathrm{H}-2$ & 10.9 & Clay-bearing nannofossil ooze & Slightly & $1.58 \mathrm{E}-02$ & $1.92 \mathrm{E}-01$ & 33 & NA & NA & NA \\
\hline $2 \mathrm{H}-3$ & 12.4 & Nannofossil marl & Slightly & $7.80 \mathrm{E}-03$ & $1.04 \mathrm{E}-01$ & 18 & NA & NA & NA \\
\hline $2 \mathrm{H}-4$ & 13.9 & Nannofossil marl & Slightly & 7.47E-02 & $8.96 \mathrm{E}-01$ & 152 & $2.30 \mathrm{E}-02$ & $2.84 \mathrm{E}-01$ & 48 \\
\hline $2 \mathrm{H}-5$ & 15.4 & Nannofossil-bearing clay & Very & $2.11 \mathrm{E}-02$ & $2.73 \mathrm{E}-01$ & 46 & $8.60 \mathrm{E}-02$ & $9.80 \mathrm{E}-01$ & 167 \\
\hline \multicolumn{10}{|c|}{ 329-U1368D- } \\
\hline 1H-1 & 1.3 & Clay-bearing nannofossil ooze & Slightly & $9.80 \mathrm{E}-03$ & $1.54 \mathrm{E}-01$ & 26 & BD & BD & BD \\
\hline $1 \mathrm{H}-2$ & 2.7 & Clay-bearing nannofossil ooze & None & $7.46 \mathrm{E}-03$ & $9.60 \mathrm{E}-02$ & 16 & $1.81 \mathrm{E}-04$ & $2.46 \mathrm{E}-03$ & 0 \\
\hline $1 \mathrm{H}-3$ & 3.8 & Clay-bearing nannofossil ooze & None & $2.51 \mathrm{E}-04$ & $3.29 \mathrm{E}-03$ & 1 & $2.11 \mathrm{E}-02$ & $3.18 \mathrm{E}-01$ & 54 \\
\hline $1 \mathrm{H}-4$ & 5.0 & Clay-bearing nannofossil ooze & None & $2.05 \mathrm{E}-04$ & $3.50 \mathrm{E}-03$ & 1 & $5.37 \mathrm{E}-03$ & $7.08 \mathrm{E}-02$ & 12 \\
\hline $1 \mathrm{H}-5$ & 7.3 & Clay-bearing nannofossil ooze & Slightly & $2.16 \mathrm{E}-02$ & $2.73 \mathrm{E}-01$ & 46 & $1.68 \mathrm{E}-03$ & $2.06 \mathrm{E}-02$ & 3 \\
\hline $2 \mathrm{H}-1$ & 8.5 & Clay-bearing nannofossil ooze & Slightly & $2.59 \mathrm{E}-02$ & $3.45 \mathrm{E}-01$ & 59 & $7.06 \mathrm{E}-04$ & $1.05 \mathrm{E}-02$ & 2 \\
\hline $2 \mathrm{H}-2$ & 9.8 & Clay-bearing nannofossil ooze & None & $2.86 \mathrm{E}-04$ & $3.53 \mathrm{E}-03$ & 1 & BD & BD & BD \\
\hline $2 \mathrm{H}-3$ & 11.2 & Nannofossil marl & None & $5.93 \mathrm{E}-03$ & $7.35 \mathrm{E}-02$ & 13 & $4.14 \mathrm{E}-02$ & $5.86 \mathrm{E}-01$ & 100 \\
\hline $2 \mathrm{H}-4$ & 13.5 & Nannofossil-bearing clay & None & $1.50 \mathrm{E}-03$ & $1.91 \mathrm{E}-02$ & 3 & $1.31 \mathrm{E}-04$ & $1.64 \mathrm{E}-03$ & 0 \\
\hline $2 \mathrm{H}-5$ & 14.8 & Nannofossil-bearing clay & None & $1.43 \mathrm{E}-02$ & $1.67 \mathrm{E}-01$ & 28 & $4.25 \mathrm{E}-04$ & $5.08 \mathrm{E}-03$ & 1 \\
\hline
\end{tabular}

$\mathrm{BD}=$ below detection, $\mathrm{NA}=$ not applicable. $\mathrm{DF}=$ drilling fluid.

Table T6. Perfluorocarbon tracer (PFT) concentration results, Holes U1369C and U1369E.

\begin{tabular}{|c|c|c|c|c|c|c|c|c|c|}
\hline \multirow{3}{*}{$\begin{array}{l}\text { Core, } \\
\text { section }\end{array}$} & \multirow{3}{*}{$\begin{array}{l}\text { Depth } \\
\text { (mbsf) }\end{array}$} & \multirow[b]{3}{*}{ Lithology } & \multirow{3}{*}{$\begin{array}{c}\text { Coring } \\
\text { disturbance } \\
\text { intensity }\end{array}$} & \multicolumn{6}{|c|}{ Contamination } \\
\hline & & & & \multicolumn{3}{|c|}{ Exterior } & \multicolumn{3}{|c|}{ Interior } \\
\hline & & & & $\left(\mathrm{ng}_{\mathrm{PFT}} / \mathrm{g}\right)$ & $\left(\mu \mathrm{L}_{\mathrm{DF}} / \mathrm{g}\right)$ & (Cells/g) & $\left(n g_{\mathrm{PFT}} / \mathrm{g}\right)$ & $\left(\mu \mathrm{L}_{\mathrm{DF}} / \mathrm{g}\right)$ & (Cells/g) \\
\hline \multicolumn{10}{|c|}{ 329-U1369C- } \\
\hline $1 \mathrm{H}-1$ & 0.2 & Metalliferous clay & None & $2.15 \mathrm{E}-04$ & $3.19 \mathrm{E}-03$ & 1 & $\mathrm{BD}$ & $\mathrm{BD}$ & $\mathrm{BD}$ \\
\hline $1 \mathrm{H}-1$ & 1.4 & Metalliferous clay & None & $6.68 \mathrm{E}-04$ & 1.19E-02 & 2 & $\mathrm{BD}$ & $\mathrm{BD}$ & $\mathrm{BD}$ \\
\hline $1 \mathrm{H}-2$ & 2.9 & Metalliferous clay & None & $5.40 \mathrm{E}-03$ & $7.85 \mathrm{E}-02$ & 13 & $6.23 \mathrm{E}-03$ & $9.60 \mathrm{E}-02$ & 16 \\
\hline $1 \mathrm{H}-3$ & 4.4 & Metalliferous clay & None & BD & BD & $\mathrm{BD}$ & $1.02 \mathrm{E}-02$ & $1.60 \mathrm{E}-01$ & 27 \\
\hline $1 \mathrm{H}-4$ & 5.8 & Metalliferous clay & None & $6.12 \mathrm{E}-03$ & $8.42 \mathrm{E}-02$ & 14 & $1.28 \mathrm{E}-02$ & $1.69 \mathrm{E}-01$ & 29 \\
\hline $2 \mathrm{H}-1$ & 7.4 & Metalliferous clay & None & $4.95 \mathrm{E}-03$ & $7.01 \mathrm{E}-02$ & 12 & $9.00 \mathrm{E}-03$ & $1.01 \mathrm{E}-01$ & 17 \\
\hline $2 \mathrm{H}-2$ & 8.9 & Metalliferous clay & None & $8.25 \mathrm{E}-03$ & $1.04 \mathrm{E}-01$ & 18 & $1.88 \mathrm{E}-02$ & $2.66 \mathrm{E}-01$ & 45 \\
\hline $2 \mathrm{H}-4$ & 11.9 & Metalliferous clay & None & BD & BD & $\mathrm{BD}$ & BD & BD & $B D$ \\
\hline $2 \mathrm{H}-5$ & 13.4 & Metalliferous clay & None & BD & BD & BD & $6.76 \mathrm{E}-03$ & $8.38 \mathrm{E}-02$ & 14 \\
\hline $2 \mathrm{H}-6$ & 14.9 & Metalliferous clay & None & NA & NA & NA & BD & BD & $\mathrm{BD}$ \\
\hline $2 \mathrm{H}-7$ & 15.7 & Metalliferous clay & None & $6.14 \mathrm{E}-03$ & 7.67E-02 & 13 & $5.60 \mathrm{E}-03$ & $8.05 \mathrm{E}-02$ & 14 \\
\hline \multicolumn{10}{|c|}{ 329-U1369E- } \\
\hline $1 \mathrm{H}-1$ & 1.5 & Metalliferous clay & None & $2.09 \mathrm{E}-02$ & $2.76 \mathrm{E}-01$ & 47 & $6.99 \mathrm{E}-04$ & $9.31 \mathrm{E}-03$ & 2 \\
\hline $1 \mathrm{H}-2$ & 2.7 & Metalliferous clay & None & $4.45 \mathrm{E}-02$ & $6.78 \mathrm{E}-01$ & 115 & NA & NA & NA \\
\hline $1 \mathrm{H}-4$ & 4.9 & Metalliferous clay & None & $3.57 \mathrm{E}-02$ & $5.49 \mathrm{E}-01$ & 93 & $4.77 \mathrm{E}-02$ & $8.31 \mathrm{E}-01$ & 141 \\
\hline $2 \mathrm{H}-1$ & 7.7 & Metalliferous clay & None & BD & BD & $\mathrm{BD}$ & $2.14 \mathrm{E}-02$ & $2.85 \mathrm{E}-01$ & 48 \\
\hline $2 \mathrm{H}-2$ & 9.6 & Metalliferous clay & None & $1.30 \mathrm{E}-02$ & $1.76 \mathrm{E}-01$ & 30 & $2.32 \mathrm{E}-02$ & $3.22 \mathrm{E}-01$ & 55 \\
\hline $2 \mathrm{H}-3$ & 10.3 & Metalliferous clay & None & $4.78 \mathrm{E}-03$ & $7.64 \mathrm{E}-02$ & 13 & NA & NA & NA \\
\hline $2 \mathrm{H}-4$ & 11.9 & Metalliferous clay & None & NA & NA & NA & $\mathrm{BD}$ & $\mathrm{BD}$ & $\mathrm{BD}$ \\
\hline $2 \mathrm{H}-5$ & 13.9 & Metalliferous clay & None & $1.78 \mathrm{E}-02$ & $2.32 \mathrm{E}-01$ & 39 & $1.58 \mathrm{E}-02$ & $2.00 \mathrm{E}-01$ & 34 \\
\hline $2 \mathrm{H}-6$ & 15.2 & Metalliferous clay & Very & $2.81 \mathrm{E}-02$ & 4.11E-01 & 70 & $1.78 \mathrm{E}-02$ & 2.64E-01 & 45 \\
\hline
\end{tabular}

$\mathrm{BD}=$ below detection, $\mathrm{NA}=$ not applicable. $\mathrm{DF}=$ drilling fluid. 
Table T7. Perfluorocarbon tracer (PFT) concentration results, Holes U1370E and U1370F. (Continued on next page.)

\begin{tabular}{|c|c|c|c|c|c|c|c|c|c|}
\hline \multirow{3}{*}{$\begin{array}{c}\text { Core, } \\
\text { section }\end{array}$} & \multirow{3}{*}{$\begin{array}{l}\text { Depth } \\
\text { (mbsf) }\end{array}$} & \multirow[b]{3}{*}{ Lithology } & \multirow{3}{*}{$\begin{array}{l}\text { Coring } \\
\text { disturbance } \\
\text { intensity }\end{array}$} & \multicolumn{6}{|c|}{ Contamination } \\
\hline & & & & & Exterior & & & Interior & \\
\hline & & & & $\left(\mathrm{ng}_{\mathrm{PFT}} / \mathrm{g}\right)$ & $\left(\mu \mathrm{L}_{\mathrm{DF}} / \mathrm{g}\right)$ & (Cells/g) & $\left(\mathrm{ng}_{\mathrm{PFT}} / \mathrm{g}\right)$ & $\left(\mu \mathrm{L}_{\mathrm{DF}} / \mathrm{g}\right)$ & (Cells/g) \\
\hline \multicolumn{10}{|c|}{ 329-U1370E- } \\
\hline $1 \mathrm{H}-1$ & 1.4 & Metalliferous clay & None & BD & $\mathrm{BD}$ & $\mathrm{BD}$ & $\mathrm{BD}$ & BD & $\mathrm{BD}$ \\
\hline $1 \mathrm{H}-2$ & 2.9 & Metalliferous clay & None & $\mathrm{BD}$ & $\mathrm{BD}$ & $\mathrm{BD}$ & $\mathrm{BD}$ & BD & $\mathrm{BD}$ \\
\hline $1 \mathrm{H}-3$ & 4.3 & Metalliferous clay & None & $1.33 \mathrm{E}-03$ & $2.68 \mathrm{E}-02$ & 5 & $\mathrm{BD}$ & $\mathrm{BD}$ & $\mathrm{BD}$ \\
\hline $1 \mathrm{H}-4$ & 6.0 & Metalliferous clay & None & $2.63 \mathrm{E}-04$ & 3.39E-03 & 1 & $2.23 \mathrm{E}-04$ & $2.78 \mathrm{E}-03$ & 0 \\
\hline $2 \mathrm{H}-1$ & 7.6 & Metalliferous clay & None & $1.66 \mathrm{E}-03$ & $2.93 \mathrm{E}-02$ & 5 & $4.55 \mathrm{E}-04$ & $1.53 \mathrm{E}-02$ & 3 \\
\hline $2 \mathrm{H}-2$ & 9.1 & Metalliferous clay & None & BD & BD & $\mathrm{BD}$ & BD & BD & BD \\
\hline $2 \mathrm{H}-3$ & 10.6 & Metalliferous clay & None & $4.33 \mathrm{E}-04$ & $6.05 \mathrm{E}-03$ & 1 & $2.91 \mathrm{E}-04$ & $3.66 \mathrm{E}-03$ & 1 \\
\hline $2 \mathrm{H}-4$ & 12.0 & Metalliferous clay & None & $5.11 \mathrm{E}-03$ & $6.75 \mathrm{E}-02$ & 11 & BD & BD & $\mathrm{BD}$ \\
\hline $2 \mathrm{H}-5$ & 13.6 & Metalliferous clay & None & BD & BD & BD & $3.17 \mathrm{E}-03$ & $4.10 \mathrm{E}-02$ & 7 \\
\hline $2 \mathrm{H}-6$ & 14.9 & Metalliferous clay & None & $\mathrm{BD}$ & $\mathrm{BD}$ & $\mathrm{BD}$ & $2.54 \mathrm{E}-04$ & $3.18 \mathrm{E}-03$ & 1 \\
\hline $3 \mathrm{H}-1$ & 17.1 & Metalliferous clay & None & $5.35 \mathrm{E}-04$ & $8.85 \mathrm{E}-03$ & 2 & BD & BD & $\mathrm{BD}$ \\
\hline $3 \mathrm{H}-2$ & 18.6 & Metalliferous clay & None & $1.24 \mathrm{E}-03$ & $1.78 \mathrm{E}-02$ & 3 & BD & BD & $\mathrm{BD}$ \\
\hline $3 \mathrm{H}-3$ & 20.1 & Metalliferous clay & None & $2.35 \mathrm{E}-04$ & $3.26 \mathrm{E}-03$ & 1 & $2.97 \mathrm{E}-03$ & $6.55 \mathrm{E}-02$ & 11 \\
\hline $3 \mathrm{H}-4$ & 21.5 & Metalliferous clay & None & 1.49E-03 & 1.99E-02 & 3 & BD & BD & $\mathrm{BD}$ \\
\hline $3 \mathrm{H}-5$ & 23.1 & Metalliferous clay & None & BD & BD & $\mathrm{BD}$ & $\mathrm{BD}$ & BD & $\mathrm{BD}$ \\
\hline $3 \mathrm{H}-6$ & 23.8 & Metalliferous clay & None & $2.59 \mathrm{E}-04$ & $3.31 \mathrm{E}-03$ & 1 & $\mathrm{BD}$ & BD & $\mathrm{BD}$ \\
\hline $4 \mathrm{H}-1$ & 26.6 & Metalliferous clay & None & $2.28 \mathrm{E}-04$ & $3.25 \mathrm{E}-03$ & 1 & $\mathrm{BD}$ & BD & $\mathrm{BD}$ \\
\hline $4 \mathrm{H}-2$ & 28.1 & Metalliferous clay & None & BD & BD & $\mathrm{BD}$ & $\mathrm{BD}$ & BD & $\mathrm{BD}$ \\
\hline $4 \mathrm{H}-3$ & 29.6 & Metalliferous clay & None & BD & $\mathrm{BD}$ & $\mathrm{BD}$ & $\mathrm{BD}$ & BD & $\mathrm{BD}$ \\
\hline $4 \mathrm{H}-5$ & 32.6 & Metalliferous clay & None & $5.62 \mathrm{E}-04$ & $8.49 \mathrm{E}-03$ & 1 & $\mathrm{BD}$ & $\mathrm{BD}$ & $\mathrm{BD}$ \\
\hline $4 \mathrm{H}-6$ & 33.2 & Metalliferous clay & None & BD & BD & BD & $\mathrm{BD}$ & BD & $\mathrm{BD}$ \\
\hline $5 \mathrm{H}-1$ & 36.1 & Metalliferous clay & None & $\mathrm{BD}$ & $\mathrm{BD}$ & $\mathrm{BD}$ & BD & $\mathrm{BD}$ & $\mathrm{BD}$ \\
\hline $5 \mathrm{H}-2$ & 37.5 & Metalliferous clay & None & $\mathrm{BD}$ & $\mathrm{BD}$ & $\mathrm{BD}$ & $2.68 \mathrm{E}-04$ & $3.20 \mathrm{E}-03$ & 1 \\
\hline $5 \mathrm{H}-3$ & 39.0 & Metalliferous clay & None & $2.56 \mathrm{E}-04$ & $4.06 \mathrm{E}-03$ & 1 & BD & BD & $\mathrm{BD}$ \\
\hline $5 \mathrm{H}-4$ & 40.4 & Metalliferous clay & None & BD & BD & $\mathrm{BD}$ & $\mathrm{BD}$ & BD & $\mathrm{BD}$ \\
\hline $5 \mathrm{H}-5$ & 42.0 & Metalliferous clay & None & $\mathrm{BD}$ & BD & $\mathrm{BD}$ & $\mathrm{BD}$ & BD & $\mathrm{BD}$ \\
\hline $5 \mathrm{H}-6$ & 42.7 & Metalliferous clay & None & $\mathrm{BD}$ & BD & $\mathrm{BD}$ & BD & $\mathrm{BD}$ & $\mathrm{BD}$ \\
\hline $6 \mathrm{H}-1$ & 45.6 & Metalliferous clay & None & $\mathrm{BD}$ & $\mathrm{BD}$ & $\mathrm{BD}$ & $3.61 \mathrm{E}-04$ & $4.72 \mathrm{E}-03$ & 1 \\
\hline $6 \mathrm{H}-2$ & 47.0 & Metalliferous clay & None & $\mathrm{BD}$ & BD & $\mathrm{BD}$ & BD & BD & $\mathrm{BD}$ \\
\hline $6 \mathrm{H}-3$ & 48.5 & Metalliferous clay & None & $\mathrm{BD}$ & $\mathrm{BD}$ & $\mathrm{BD}$ & $\mathrm{BD}$ & $\mathrm{BD}$ & $\mathrm{BD}$ \\
\hline $6 \mathrm{H}-4$ & 50.0 & Metalliferous clay & None & 4.45E-04 & $5.22 \mathrm{E}-03$ & 1 & $1.09 \mathrm{E}-04$ & $1.51 \mathrm{E}-03$ & 0 \\
\hline $6 \mathrm{H}-5$ & 51.1 & Metalliferous clay & Slurry & BD & BD & BD & BD & BD & $\mathrm{BD}$ \\
\hline $8 \mathrm{H}-2$ & 58.3 & Metalliferous clay & Very & $4.34 \mathrm{E}-04$ & $5.20 \mathrm{E}-03$ & 1 & BD & BD & $\mathrm{BD}$ \\
\hline $8 \mathrm{H}-3$ & 59.8 & Metalliferous clay & Very & BD & BD & $\mathrm{BD}$ & $3.70 \mathrm{E}-04$ & $5.40 \mathrm{E}-03$ & 1 \\
\hline $8 \mathrm{H}-4$ & 61.2 & Metalliferous clay & Very & BD & $\mathrm{BD}$ & $\mathrm{BD}$ & BD & BD & $\mathrm{BD}$ \\
\hline $8 \mathrm{H}-5$ & 62.8 & Pelagic clay & Very & $\mathrm{BD}$ & $\mathrm{BD}$ & $\mathrm{BD}$ & $1.58 \mathrm{E}-03$ & $2.00 \mathrm{E}-02$ & 3 \\
\hline $9 \mathrm{H}-1$ & 63.5 & Pelagic clay & Very & BD & BD & $\mathrm{BD}$ & $5.96 \mathrm{E}-04$ & $7.08 \mathrm{E}-03$ & 1 \\
\hline $8 \mathrm{H}-6$ & 64.3 & Pelagic clay & Very & $2.80 \mathrm{E}-04$ & $4.23 \mathrm{E}-03$ & 1 & BD & BD & $\mathrm{BD}$ \\
\hline $9 \mathrm{H}-2$ & 65.0 & Pelagic clay & Very & NA & NA & NA & BD & BD & BD \\
\hline \multicolumn{10}{|c|}{ 329-U1370F- } \\
\hline $1 \mathrm{H}-1$ & 1.5 & Metalliferous clay & None & 3.87E-04 & $6.41 \mathrm{E}-03$ & 1 & $\mathrm{BD}$ & BD & $\mathrm{BD}$ \\
\hline $1 \mathrm{H}-2$ & 3.0 & Metalliferous clay & None & BD & $\mathrm{BD}$ & $\mathrm{BD}$ & $\mathrm{BD}$ & $\mathrm{BD}$ & $\mathrm{BD}$ \\
\hline $1 \mathrm{H}-3$ & 4.1 & Metalliferous clay & None & $4.42 \mathrm{E}-03$ & $6.10 \mathrm{E}-02$ & 10 & $\mathrm{BD}$ & $\mathrm{BD}$ & $\mathrm{BD}$ \\
\hline $1 \mathrm{H}-4$ & 5.1 & Metalliferous clay & None & $8.96 \mathrm{E}-03$ & $1.35 \mathrm{E}-01$ & 23 & $\mathrm{BD}$ & $\mathrm{BD}$ & $\mathrm{BD}$ \\
\hline $2 \mathrm{H}-1$ & 7.9 & Metalliferous clay & None & $1.93 \mathrm{E}-03$ & $2.56 \mathrm{E}-02$ & 4 & $\mathrm{BD}$ & $\mathrm{BD}$ & $\mathrm{BD}$ \\
\hline $2 \mathrm{H}-3$ & 10.7 & Metalliferous clay & None & $6.62 \mathrm{E}-04$ & $9.09 \mathrm{E}-03$ & 2 & $\mathrm{BD}$ & $\mathrm{BD}$ & $\mathrm{BD}$ \\
\hline $2 \mathrm{H}-5$ & 13.1 & Metalliferous clay & None & BD & BD & $\mathrm{BD}$ & $\mathrm{BD}$ & $\mathrm{BD}$ & $\mathrm{BD}$ \\
\hline $3 \mathrm{H}-2$ & 18.6 & Metalliferous clay & None & $\mathrm{BD}$ & $\mathrm{BD}$ & $\mathrm{BD}$ & $\mathrm{BD}$ & BD & $\mathrm{BD}$ \\
\hline $3 \mathrm{H}-5$ & 22.7 & Metalliferous clay & None & $\mathrm{BD}$ & $\mathrm{BD}$ & $\mathrm{BD}$ & $\mathrm{BD}$ & $\mathrm{BD}$ & $\mathrm{BD}$ \\
\hline $4 \mathrm{H}-3$ & 29.8 & Metalliferous clay & None & $\mathrm{BD}$ & $\mathrm{BD}$ & $\mathrm{BD}$ & $\mathrm{BD}$ & $\mathrm{BD}$ & $\mathrm{BD}$ \\
\hline $4 \mathrm{H}-5$ & 32.2 & Metalliferous clay & None & $\mathrm{BD}$ & $\mathrm{BD}$ & $\mathrm{BD}$ & $\mathrm{BD}$ & $\mathrm{BD}$ & $\mathrm{BD}$ \\
\hline $5 \mathrm{H}-2$ & 37.9 & Metalliferous clay & None & $\mathrm{BD}$ & $\mathrm{BD}$ & $\mathrm{BD}$ & $\mathrm{BD}$ & $\mathrm{BD}$ & $\mathrm{BD}$ \\
\hline $5 \mathrm{H}-3$ & 39.6 & Metalliferous clay & None & BD & BD & BD & BD & BD & BD \\
\hline $5 \mathrm{H}-4$ & 41.1 & Metalliferous clay & None & $\mathrm{BD}$ & $\mathrm{BD}$ & $\mathrm{BD}$ & $\mathrm{BD}$ & BD & $\mathrm{BD}$ \\
\hline $5 \mathrm{H}-5$ & 42.0 & Metalliferous clay & None & BD & $\mathrm{BD}$ & BD & BD & BD & BD \\
\hline $5 \mathrm{H}-5$ & 42.6 & Metalliferous clay & None & $\mathrm{BD}$ & $\mathrm{BD}$ & $\mathrm{BD}$ & $\mathrm{BD}$ & $\mathrm{BD}$ & $\mathrm{BD}$ \\
\hline $6 \mathrm{H}-2$ & 47.6 & Metalliferous clay & None & $\mathrm{BD}$ & $\mathrm{BD}$ & $\mathrm{BD}$ & $\mathrm{BD}$ & $\mathrm{BD}$ & $\mathrm{BD}$ \\
\hline $6 \mathrm{H}-3$ & 48.7 & Metalliferous clay & None & $\mathrm{BD}$ & $\mathrm{BD}$ & $\mathrm{BD}$ & $\mathrm{BD}$ & $\mathrm{BD}$ & $\mathrm{BD}$ \\
\hline $6 \mathrm{H}-3$ & 49.1 & Metalliferous clay & None & $\mathrm{BD}$ & $\mathrm{BD}$ & $\mathrm{BD}$ & $\mathrm{BD}$ & $\mathrm{BD}$ & $\mathrm{BD}$ \\
\hline $6 \mathrm{H}-4$ & 50.6 & Metalliferous clay & None & $\mathrm{BD}$ & $\mathrm{BD}$ & $\mathrm{BD}$ & $\mathrm{BD}$ & $\mathrm{BD}$ & $\mathrm{BD}$ \\
\hline $6 \mathrm{H}-5$ & 51.1 & Metalliferous clay & None & $\mathrm{BD}$ & $\mathrm{BD}$ & $\mathrm{BD}$ & NA & NA & NA \\
\hline $6 \mathrm{H}-5$ & 52.1 & Metalliferous clay & None & BD & BD & $\mathrm{BD}$ & BD & BD & $\mathrm{BD}$ \\
\hline $7 \mathrm{H}-1$ & 55.6 & Metalliferous clay & None & $\mathrm{BD}$ & BD & $\mathrm{BD}$ & $\mathrm{BD}$ & BD & $\mathrm{BD}$ \\
\hline $7 \mathrm{H}-2$ & 56.8 & Metalliferous clay & None & $\mathrm{BD}$ & $\mathrm{BD}$ & $\mathrm{BD}$ & $\mathrm{BD}$ & $\mathrm{BD}$ & $\mathrm{BD}$ \\
\hline $7 \mathrm{H}-2$ & 57.1 & Metalliferous clay & None & $B D$ & $\mathrm{BD}$ & $\mathrm{BD}$ & $\mathrm{BD}$ & $B D$ & $\mathrm{BD}$ \\
\hline
\end{tabular}


Table T7 (continued).

\begin{tabular}{|c|c|c|c|c|c|c|c|c|c|}
\hline \multirow{3}{*}{$\begin{array}{l}\text { Core, } \\
\text { section }\end{array}$} & \multirow{3}{*}{$\begin{array}{l}\text { Depth } \\
\text { (mbsf) }\end{array}$} & \multirow[b]{3}{*}{ Lithology } & \multirow{3}{*}{$\begin{array}{c}\text { Coring } \\
\text { disturbance } \\
\text { intensity }\end{array}$} & \multicolumn{6}{|c|}{ Contamination } \\
\hline & & & & \multicolumn{3}{|c|}{ Exterior } & \multicolumn{3}{|c|}{ Interior } \\
\hline & & & & $\left(n g_{P F T} / g\right)$ & $\left(\mu \mathrm{L}_{\mathrm{DF}} / \mathrm{g}\right)$ & (Cells/g) & $\left(n g_{P F T} / g\right)$ & $\left(\mu \mathrm{L}_{\mathrm{DF}} / \mathrm{g}\right)$ & (Cells/g) \\
\hline $7 \mathrm{H}-3$ & 57.7 & Metalliferous clay & None & BD & BD & $\mathrm{BD}$ & $4.04 \mathrm{E}-03$ & $5.00 \mathrm{E}-02$ & 8 \\
\hline $7 \mathrm{H}-3$ & 58.6 & Metalliferous clay & None & BD & BD & BD & BD & BD & BD \\
\hline $7 \mathrm{H}-4$ & 59.6 & Metalliferous clay & None & BD & BD & BD & BD & $\mathrm{BD}$ & BD \\
\hline $7 \mathrm{H}-4$ & 60.0 & Metalliferous clay & None & BD & BD & BD & BD & $\mathrm{BD}$ & BD \\
\hline $7 \mathrm{H}-5$ & 61.1 & Metalliferous clay & None & BD & BD & BD & BD & BD & BD \\
\hline $7 \mathrm{H}-5$ & 61.6 & Metalliferous clay & None & $\mathrm{BD}$ & BD & BD & BD & BD & BD \\
\hline $7 \mathrm{H}-6$ & 63.1 & Metalliferous clay & None & BD & BD & BD & $3.10 \mathrm{E}-05$ & $3.86 \mathrm{E}-04$ & $\mathrm{BD}$ \\
\hline $8 \mathrm{H}-1$ & 64.7 & Metalliferous clay & Very & $8.81 \mathrm{E}-03$ & $1.13 \mathrm{E}-01$ & 19 & BD & BD & $\mathrm{BD}$ \\
\hline
\end{tabular}

$\mathrm{BD}=$ below detection, $\mathrm{NA}=$ not applicable. $\mathrm{DF}=$ drilling fluid.

Table T8. Perfluorocarbon tracer (PFT) concentration results, Holes U1371E and U1371F. (Continued on next page.)

\begin{tabular}{|c|c|c|c|c|c|c|c|c|c|}
\hline \multirow{3}{*}{$\begin{array}{c}\text { Core, } \\
\text { section }\end{array}$} & \multirow{3}{*}{$\begin{array}{l}\text { Depth } \\
\text { (mbsf) }\end{array}$} & \multirow[b]{3}{*}{ Lithology } & \multirow{3}{*}{$\begin{array}{c}\text { Coring } \\
\text { disturbance } \\
\text { intensity }\end{array}$} & \multicolumn{6}{|c|}{ Contamination } \\
\hline & & & & & Exterior & & & Interior & \\
\hline & & & & $\left(\mathrm{ng}_{\mathrm{PFT}} / \mathrm{g}\right)$ & $\left(\mu \mathrm{L}_{\mathrm{DF}} / \mathrm{g}\right)$ & (Cells/g) & $\left(\mathrm{ng}_{\mathrm{PFT}} / \mathrm{g}\right)$ & $\left(\mu \mathrm{L}_{\mathrm{DF}} / \mathrm{g}\right)$ & (Cells/g) \\
\hline \multicolumn{10}{|c|}{ 329-U1371E- } \\
\hline $1 \mathrm{H}-1$ & 1.4 & Diatom ooze & None & $\mathrm{BD}$ & $\mathrm{BD}$ & $\mathrm{BD}$ & $5.24 \mathrm{E}-04$ & $7.05 \mathrm{E}-03$ & 1 \\
\hline $1 \mathrm{H}-2$ & 2.9 & Diatom ooze & None & BD & $B D$ & $\mathrm{BD}$ & BD & BD & $\mathrm{BD}$ \\
\hline $1 \mathrm{H}-3$ & 4.3 & Diatom ooze & None & BD & $\mathrm{BD}$ & $\mathrm{BD}$ & $\mathrm{BD}$ & $\mathrm{BD}$ & $\mathrm{BD}$ \\
\hline $1 \mathrm{H}-4$ & 5.9 & Diatom ooze & None & BD & $\mathrm{BD}$ & $\mathrm{BD}$ & $\mathrm{BD}$ & $\mathrm{BD}$ & $\mathrm{BD}$ \\
\hline $1 \mathrm{H}-5$ & 7.4 & Diatom ooze & None & $\mathrm{BD}$ & $\mathrm{BD}$ & $B D$ & $\mathrm{BD}$ & $\mathrm{BD}$ & $\mathrm{BD}$ \\
\hline $1 \mathrm{H}-6$ & 8.0 & Diatom ooze & None & $\mathrm{BD}$ & $\mathrm{BD}$ & $\mathrm{BD}$ & $\mathrm{BD}$ & $\mathrm{BD}$ & $\mathrm{BD}$ \\
\hline $2 \mathrm{H}-1$ & 9.6 & Diatom ooze & None & $\mathrm{BD}$ & $\mathrm{BD}$ & $\mathrm{BD}$ & $\mathrm{BD}$ & $\mathrm{BD}$ & $\mathrm{BD}$ \\
\hline $2 \mathrm{H}-2$ & 11.1 & Diatom ooze & None & BD & $\mathrm{BD}$ & $\mathrm{BD}$ & $\mathrm{BD}$ & $\mathrm{BD}$ & $\mathrm{BD}$ \\
\hline $2 \mathrm{H}-3$ & 12.6 & Diatom ooze & None & $\mathrm{BD}$ & $B D$ & $\mathrm{BD}$ & $\mathrm{BD}$ & $B D$ & $\mathrm{BD}$ \\
\hline $2 \mathrm{H}-4$ & 14.1 & Diatom ooze & None & $\mathrm{BD}$ & $\mathrm{BD}$ & $\mathrm{BD}$ & $\mathrm{BD}$ & $\mathrm{BD}$ & $\mathrm{BD}$ \\
\hline $2 \mathrm{H}-5$ & 15.1 & Diatom ooze & None & $\mathrm{BD}$ & $\mathrm{BD}$ & $\mathrm{BD}$ & $\mathrm{BD}$ & $\mathrm{BD}$ & $\mathrm{BD}$ \\
\hline $3 \mathrm{H}-2$ & 20.6 & Diatom ooze & None & BD & $\mathrm{BD}$ & $\mathrm{BD}$ & $\mathrm{BD}$ & $\mathrm{BD}$ & $\mathrm{BD}$ \\
\hline $3 \mathrm{H}-3$ & 22.0 & Diatom ooze & None & $\mathrm{BD}$ & $B D$ & $\mathrm{BD}$ & $\mathrm{BD}$ & $\mathrm{BD}$ & $\mathrm{BD}$ \\
\hline $3 \mathrm{H}-4$ & 23.6 & Diatom ooze & None & $\mathrm{BD}$ & $B D$ & $\mathrm{BD}$ & $B D$ & $\mathrm{BD}$ & $\mathrm{BD}$ \\
\hline $3 \mathrm{H}-5$ & 24.9 & Diatom ooze & None & $\mathrm{BD}$ & $\mathrm{BD}$ & $B D$ & $B D$ & $\mathrm{BD}$ & $\mathrm{BD}$ \\
\hline $3 \mathrm{H}-6$ & 25.4 & Diatom ooze & None & $\mathrm{BD}$ & $B D$ & $\mathrm{BD}$ & $B D$ & $\mathrm{BD}$ & $\mathrm{BD}$ \\
\hline $4 \mathrm{H}-1$ & 28.6 & Diatom ooze & None & $\mathrm{BD}$ & $\mathrm{BD}$ & $\mathrm{BD}$ & NA & NA & NA \\
\hline $4 \mathrm{H}-2$ & 30.1 & Diatom ooze & None & $\mathrm{BD}$ & $\mathrm{BD}$ & $\mathrm{BD}$ & $\mathrm{BD}$ & BD & $\mathrm{BD}$ \\
\hline $4 \mathrm{H}-3$ & 31.6 & Diatom ooze & None & BD & $\mathrm{BD}$ & $\mathrm{BD}$ & $\mathrm{BD}$ & $B D$ & $\mathrm{BD}$ \\
\hline $4 \mathrm{H}-4$ & 33.1 & Diatom ooze & None & $\mathrm{BD}$ & $B D$ & $\mathrm{BD}$ & $\mathrm{BD}$ & $B D$ & $\mathrm{BD}$ \\
\hline $4 \mathrm{H}-5$ & 34.4 & Diatom ooze & None & $\mathrm{BD}$ & $\mathrm{BD}$ & $\mathrm{BD}$ & $\mathrm{BD}$ & $\mathrm{BD}$ & $\mathrm{BD}$ \\
\hline $5 \mathrm{H}-1$ & 38.1 & Diatom ooze & None & $\mathrm{BD}$ & $\mathrm{BD}$ & $\mathrm{BD}$ & $B D$ & $B D$ & $\mathrm{BD}$ \\
\hline $5 \mathrm{H}-2$ & 39.6 & Diatom ooze & None & $\mathrm{BD}$ & $\mathrm{BD}$ & $\mathrm{BD}$ & $B D$ & $\mathrm{BD}$ & $\mathrm{BD}$ \\
\hline $5 \mathrm{H}-3$ & 41.1 & Diatom ooze & None & BD & $\mathrm{BD}$ & $\mathrm{BD}$ & $\mathrm{BD}$ & $\mathrm{BD}$ & $\mathrm{BD}$ \\
\hline $5 \mathrm{H}-4$ & 42.6 & Diatom ooze & None & $\mathrm{BD}$ & $B D$ & $\mathrm{BD}$ & $\mathrm{BD}$ & $B D$ & $\mathrm{BD}$ \\
\hline $5 \mathrm{H}-5$ & 43.3 & Diatom ooze & None & $\mathrm{BD}$ & $\mathrm{BD}$ & $\mathrm{BD}$ & $\mathrm{BD}$ & $\mathrm{BD}$ & $\mathrm{BD}$ \\
\hline $6 \mathrm{H}-3$ & 50.6 & Diatom ooze & Very & $\mathrm{BD}$ & $B D$ & $\mathrm{BD}$ & $\mathrm{BD}$ & $\mathrm{BD}$ & $\mathrm{BD}$ \\
\hline $6 \mathrm{H}-5$ & 53.6 & Diatom ooze & None & $\mathrm{BD}$ & $\mathrm{BD}$ & $\mathrm{BD}$ & $\mathrm{BD}$ & $\mathrm{BD}$ & $\mathrm{BD}$ \\
\hline 7H-1 & 57.1 & Diatom ooze & None & $\mathrm{BD}$ & $\mathrm{BD}$ & $\mathrm{BD}$ & $\mathrm{BD}$ & $\mathrm{BD}$ & $\mathrm{BD}$ \\
\hline $7 \mathrm{H}-2$ & 58.6 & Diatom ooze & None & BD & $\mathrm{BD}$ & $\mathrm{BD}$ & $B D$ & $\mathrm{BD}$ & $\mathrm{BD}$ \\
\hline $7 \mathrm{H}-3$ & 60.0 & Diatom ooze & None & $\mathrm{BD}$ & $\mathrm{BD}$ & $\mathrm{BD}$ & $\mathrm{BD}$ & $\mathrm{BD}$ & $\mathrm{BD}$ \\
\hline $7 \mathrm{H}-4$ & 61.7 & Diatom ooze & None & $\mathrm{BD}$ & $\mathrm{BD}$ & $\mathrm{BD}$ & $\mathrm{BD}$ & $\mathrm{BD}$ & $\mathrm{BD}$ \\
\hline $7 \mathrm{H}-5$ & 63.1 & Diatom ooze & None & BD & $\mathrm{BD}$ & $\mathrm{BD}$ & $\mathrm{BD}$ & $\mathrm{BD}$ & $\mathrm{BD}$ \\
\hline $7 \mathrm{H}-6$ & 64.5 & Diatom ooze & None & $\mathrm{BD}$ & $\mathrm{BD}$ & $\mathrm{BD}$ & $\mathrm{BD}$ & $\mathrm{BD}$ & $\mathrm{BD}$ \\
\hline $8 \mathrm{H}-1$ & 66.6 & Diatom ooze & Very & $\mathrm{BD}$ & $\mathrm{BD}$ & $\mathrm{BD}$ & $\mathrm{BD}$ & $B D$ & $\mathrm{BD}$ \\
\hline $8 \mathrm{H}-3$ & 69.6 & Diatom ooze & None & $\mathrm{BD}$ & $\mathrm{BD}$ & $B D$ & $B D$ & $\mathrm{BD}$ & $\mathrm{BD}$ \\
\hline $8 \mathrm{H}-4$ & 70.5 & Diatom ooze & None & $\mathrm{BD}$ & $\mathrm{BD}$ & $\mathrm{BD}$ & $\mathrm{BD}$ & $B D$ & $\mathrm{BD}$ \\
\hline $8 \mathrm{H}-5$ & 72.6 & Diatom ooze & None & $\mathrm{BD}$ & $\mathrm{BD}$ & $\mathrm{BD}$ & $\mathrm{BD}$ & $\mathrm{BD}$ & $\mathrm{BD}$ \\
\hline $8 \mathrm{H}-6$ & 73.5 & Diatom ooze & None & $\mathrm{BD}$ & $\mathrm{BD}$ & $\mathrm{BD}$ & $\mathrm{BD}$ & $B D$ & $\mathrm{BD}$ \\
\hline $9 \mathrm{H}-2$ & 76.5 & Diatom ooze & None & $\mathrm{BD}$ & $\mathrm{BD}$ & $\mathrm{BD}$ & $\mathrm{BD}$ & $B D$ & $\mathrm{BD}$ \\
\hline $9 \mathrm{H}-3$ & 77.4 & Diatom ooze & None & $\mathrm{BD}$ & $B D$ & $\mathrm{BD}$ & $\mathrm{BD}$ & $B D$ & $\mathrm{BD}$ \\
\hline $9 \mathrm{H}-4$ & 79.5 & Diatom ooze & None & $\mathrm{BD}$ & $\mathrm{BD}$ & $\mathrm{BD}$ & $\mathrm{BD}$ & $B D$ & $\mathrm{BD}$ \\
\hline $9 \mathrm{H}-5$ & 80.4 & Diatom ooze & None & $\mathrm{BD}$ & $\mathrm{BD}$ & $\mathrm{BD}$ & $B D$ & $B D$ & $\mathrm{BD}$ \\
\hline $9 \mathrm{H}-6$ & 82.5 & Diatom ooze & None & $\mathrm{BD}$ & $\mathrm{BD}$ & $\mathrm{BD}$ & $B D$ & $\mathrm{BD}$ & $\mathrm{BD}$ \\
\hline $9 \mathrm{H}-7$ & 83.2 & Diatom ooze & None & $\mathrm{BD}$ & $\mathrm{BD}$ & $\mathrm{BD}$ & $\mathrm{BD}$ & $\mathrm{BD}$ & $\mathrm{BD}$ \\
\hline $10 \mathrm{H}-1$ & 85.6 & Diatom ooze & None & $\mathrm{BD}$ & $B D$ & $\mathrm{BD}$ & BD & $\mathrm{BD}$ & $\mathrm{BD}$ \\
\hline $10 \mathrm{H}-2$ & 86.7 & Diatom ooze & None & BD & $\mathrm{BD}$ & BD & $3.29 \mathrm{E}-04$ & $4.67 \mathrm{E}-03$ & 1 \\
\hline $10 \mathrm{H}-3$ & 88.6 & Diatom ooze & None & $\mathrm{BD}$ & $\mathrm{BD}$ & $\mathrm{BD}$ & BD & BD & $\mathrm{BD}$ \\
\hline
\end{tabular}


Table T8 (continued).

\begin{tabular}{|c|c|c|c|c|c|c|c|c|c|}
\hline \multirow{3}{*}{$\begin{array}{c}\text { Core, } \\
\text { section }\end{array}$} & \multirow{3}{*}{$\begin{array}{l}\text { Depth } \\
\text { (mbsf) }\end{array}$} & \multirow[b]{3}{*}{ Lithology } & \multirow{3}{*}{$\begin{array}{c}\text { Coring } \\
\text { disturbance } \\
\text { intensity }\end{array}$} & \multicolumn{6}{|c|}{ Contamination } \\
\hline & & & & \multicolumn{3}{|c|}{ Exterior } & \multicolumn{3}{|c|}{ Interior } \\
\hline & & & & $\left(\mathrm{ng}_{\mathrm{PFT}} / \mathrm{g}\right)$ & $\left(\mu \mathrm{L}_{\mathrm{DF}} / \mathrm{g}\right)$ & (Cells/g) & $\left(\mathrm{ng}_{\mathrm{PFT}} / \mathrm{g}\right)$ & $\left(\mu \mathrm{L}_{\mathrm{DF}} / \mathrm{g}\right)$ & (Cells/g) \\
\hline $10 \mathrm{H}-4$ & 89.5 & Diatom ooze & None & $\mathrm{BD}$ & BD & BD & BD & $\mathrm{BD}$ & $\mathrm{BD}$ \\
\hline $10 \mathrm{H}-5$ & 91.6 & Diatom ooze & None & $B D$ & $B D$ & $\mathrm{BD}$ & $B D$ & $\mathrm{BD}$ & $\mathrm{BD}$ \\
\hline $10 \mathrm{H}-6$ & 92.7 & Diatom ooze & None & $B D$ & $B D$ & $\mathrm{BD}$ & $\mathrm{BD}$ & $\mathrm{BD}$ & $\mathrm{BD}$ \\
\hline $11 \mathrm{H}-1$ & 95.1 & Diatom ooze & None & $B D$ & $B D$ & $\mathrm{BD}$ & $B D$ & $\mathrm{BD}$ & $\mathrm{BD}$ \\
\hline $11 \mathrm{H}-2$ & 96.0 & Diatom ooze & None & NA & NA & NA & BD & $\mathrm{BD}$ & $\mathrm{BD}$ \\
\hline $11 \mathrm{H}-3$ & 98.0 & Diatom ooze & None & $4.04 \mathrm{E}-04$ & $5.09 \mathrm{E}-03$ & 1 & $B D$ & $\mathrm{BD}$ & $\mathrm{BD}$ \\
\hline $11 \mathrm{H}-4$ & 98.9 & Diatom ooze & None & BD & BD & $\mathrm{BD}$ & BD & $\mathrm{BD}$ & $\mathrm{BD}$ \\
\hline $11 \mathrm{H}-5$ & 101.1 & Diatom ooze & None & $\mathrm{BD}$ & $B D$ & $\mathrm{BD}$ & $B D$ & $\mathrm{BD}$ & $\mathrm{BD}$ \\
\hline $11 \mathrm{H}-6$ & 102.2 & Diatom ooze & None & $B D$ & $B D$ & $\mathrm{BD}$ & $B D$ & $\mathrm{BD}$ & $\mathrm{BD}$ \\
\hline $12 \mathrm{H}-2$ & 106.1 & Diatom ooze & None & BD & BD & $\mathrm{BD}$ & $B D$ & $\mathrm{BD}$ & $\mathrm{BD}$ \\
\hline $12 \mathrm{H}-3$ & 107.5 & Diatom ooze & None & $\mathrm{BD}$ & $B D$ & $\mathrm{BD}$ & $B D$ & $\mathrm{BD}$ & $\mathrm{BD}$ \\
\hline $12 \mathrm{H}-4$ & 108.8 & Diatom ooze & None & $\mathrm{BD}$ & $B D$ & $\mathrm{BD}$ & $B D$ & $\mathrm{BD}$ & $\mathrm{BD}$ \\
\hline $13 \mathrm{H}-1$ & 114.1 & Zeolitic metalliferous clay & None & $\mathrm{BD}$ & $\mathrm{BD}$ & $\mathrm{BD}$ & BD & $\mathrm{BD}$ & $\mathrm{BD}$ \\
\hline $13 \mathrm{H}-2$ & 115.6 & Zeolitic metalliferous clay & None & $\mathrm{BD}$ & $\mathrm{BD}$ & $\mathrm{BD}$ & $B D$ & $\mathrm{BD}$ & $\mathrm{BD}$ \\
\hline $13 \mathrm{H}-3$ & 117.0 & Zeolitic metalliferous clay & None & $\mathrm{BD}$ & $\mathrm{BD}$ & $\mathrm{BD}$ & BD & $\mathrm{BD}$ & $\mathrm{BD}$ \\
\hline $13 \mathrm{H}-4$ & 117.7 & Zeolitic metalliferous clay & None & $\mathrm{BD}$ & $B D$ & $\mathrm{BD}$ & BD & $\mathrm{BD}$ & $\mathrm{BD}$ \\
\hline $13 \mathrm{H}-4$ & 118.6 & Zeolitic metalliferous clay & None & $\mathrm{BD}$ & $B D$ & $\mathrm{BD}$ & BD & $\mathrm{BD}$ & $\mathrm{BD}$ \\
\hline $13 \mathrm{H}-5$ & 120.1 & Metalliferous clay & None & $\mathrm{BD}$ & $B D$ & $\mathrm{BD}$ & BD & $\mathrm{BD}$ & $\mathrm{BD}$ \\
\hline $13 \mathrm{H}-6$ & 121.6 & Metalliferous clay & None & $\mathrm{BD}$ & $B D$ & $\mathrm{BD}$ & $B D$ & $\mathrm{BD}$ & $\mathrm{BD}$ \\
\hline $13 \mathrm{H}-7$ & 122.0 & Metalliferous clay & None & $\mathrm{BD}$ & $B D$ & $\mathrm{BD}$ & $B D$ & $\mathrm{BD}$ & $\mathrm{BD}$ \\
\hline $14 \mathrm{H}-1$ & 123.6 & Metalliferous clay & None & $\mathrm{BD}$ & $B D$ & $\mathrm{BD}$ & $B D$ & $\mathrm{BD}$ & $\mathrm{BD}$ \\
\hline $14 \mathrm{H}-2$ & 125.1 & Metalliferous clay & None & $\mathrm{BD}$ & $B D$ & $\mathrm{BD}$ & $B D$ & $\mathrm{BD}$ & $\mathrm{BD}$ \\
\hline $14 \mathrm{H}-3$ & 126.5 & Metalliferous clay & None & $\mathrm{BD}$ & BD & $\mathrm{BD}$ & BD & $\mathrm{BD}$ & $\mathrm{BD}$ \\
\hline $14 \mathrm{H}-4$ & 128.0 & Metalliferous clay & None & $\mathrm{BD}$ & $B D$ & $\mathrm{BD}$ & BD & $\mathrm{BD}$ & $\mathrm{BD}$ \\
\hline $14 \mathrm{H}-5$ & 129.5 & Metalliferous clay & Very & BD & BD & $\mathrm{BD}$ & $\mathrm{BD}$ & $\mathrm{BD}$ & $\mathrm{BD}$ \\
\hline $14 \mathrm{H}-6$ & 130.2 & Metalliferous clay & Very & $\mathrm{BD}$ & BD & $\mathrm{BD}$ & NA & NA & NA \\
\hline \multicolumn{10}{|c|}{ 329-U1371F- } \\
\hline $1 \mathrm{H}-1$ & 1.4 & Diatom ooze & None & BD & $\mathrm{BD}$ & BD & $\mathrm{BD}$ & $\mathrm{BD}$ & $\mathrm{BD}$ \\
\hline $1 \mathrm{H}-2$ & 2.8 & Diatom ooze & None & $\mathrm{BD}$ & $B D$ & $\mathrm{BD}$ & $\mathrm{BD}$ & $\mathrm{BD}$ & $\mathrm{BD}$ \\
\hline $1 \mathrm{H}-3$ & 4.1 & Diatom ooze & None & $\mathrm{BD}$ & $B D$ & $\mathrm{BD}$ & $B D$ & $\mathrm{BD}$ & $\mathrm{BD}$ \\
\hline $1 \mathrm{H}-4$ & 5.2 & Diatom ooze & None & $\mathrm{BD}$ & $B D$ & $\mathrm{BD}$ & $B D$ & $\mathrm{BD}$ & $\mathrm{BD}$ \\
\hline $1 \mathrm{H}-5$ & 6.6 & Diatom ooze & None & $\mathrm{BD}$ & $B D$ & $\mathrm{BD}$ & $B D$ & $\mathrm{BD}$ & $\mathrm{BD}$ \\
\hline $2 \mathrm{H}-2$ & 11.8 & Diatom ooze & None & $\mathrm{BD}$ & $B D$ & $\mathrm{BD}$ & $B D$ & $\mathrm{BD}$ & $\mathrm{BD}$ \\
\hline $2 \mathrm{H}-4$ & 14.1 & Diatom ooze & None & BD & $B D$ & $\mathrm{BD}$ & BD & $\mathrm{BD}$ & $\mathrm{BD}$ \\
\hline $14 \mathrm{H}-3$ & 126.9 & Metalliferous clay & None & $\mathrm{BD}$ & $B D$ & $\mathrm{BD}$ & $\mathrm{BD}$ & $\mathrm{BD}$ & $\mathrm{BD}$ \\
\hline $14 \mathrm{H}-4$ & 127.8 & Metalliferous clay & None & $\mathrm{BD}$ & $B D$ & $\mathrm{BD}$ & NA & NA & NA \\
\hline $14 \mathrm{H}-4$ & 128.9 & Metalliferous clay & None & $\mathrm{BD}$ & $B D$ & $\mathrm{BD}$ & $\mathrm{BD}$ & $\mathrm{BD}$ & $\mathrm{BD}$ \\
\hline
\end{tabular}

$\mathrm{BD}=$ below detection, $\mathrm{NA}=$ not applicable $\mathrm{DF}=$ drilling fluid. 


\section{Appendix A}

\section{Preparation of standards for gas chromatograph calibration}

1. PFT dilution series in methanol $(\mathrm{MEOH})$ :

$$
\begin{aligned}
& 10^{-2}=9.9 \mathrm{~mL} \mathrm{MEOH}+0.1 \mathrm{~mL} \mathrm{PFT} \\
& 10^{-4}=9.9 \mathrm{~mL} \mathrm{MEOH}+0.1 \mathrm{~mL} 10^{-2} \mathrm{PFT} \\
& 10^{-6}=9.9 \mathrm{~mL} \mathrm{MEOH}+0.1 \mathrm{~mL} 10^{-4} \mathrm{PFT} \\
& 10^{-8}=9.9 \mathrm{~mL} \mathrm{MEOH}+0.1 \mathrm{~mL} 10^{-6} \mathrm{PFT}
\end{aligned}
$$

2. Transfer $10 \mu \mathrm{L}$ aliquots of each dilution into duplicate $20 \mathrm{~mL}$ capacity headspace vials, seal each with a polytetrafluoroethylene (PTFE)/silica septum, and label appropriately.

3. Heat the vials at $70^{\circ} \mathrm{C}$ for $30 \mathrm{~min}$ along with the $3 \mathrm{~m}$ gas-tight plastic syringes and needles that will be used for analysis. A rotation of 4 needles may be used, provided that each is allocated a 30 min heating period between sequential usages.

4. Inject either $0.5,1.0$, or $2.0 \mathrm{~mL}$ of each standard's headspace gas into the gas chromatograph on separate runs using the $3 \mathrm{~mL}$ plastic syringe affixed with a luer fitting for stainless steel needles.

5. Integrate the peak area for each injection. Convert the PFT:MEOH dilution to PFT(g) using the following formula:

$$
\operatorname{PFT}(\mathrm{g})=V_{\text {injected }} \times C_{\text {std }}
$$

$$
\begin{array}{ll}
\text { where } & \\
V_{\text {injected }}= & \text { standard volume injected, } \\
C_{\text {std }} & \rho_{\text {PFT }} \times \mathrm{df} \times V_{\text {STD_solution }} / V_{\text {headspace }} \text { in } \\
& \text { grams PFT/volume headspace, } \\
= & \text { density of PFT (i.e., } 1.78 \mathrm{~g} / \mathrm{L}), \\
\rho_{\text {PFT }} & \text { dilution factor, ratio }(\mathrm{vol}: \mathrm{vol}) \text { of } \\
\mathrm{df} & \text { pure PFT:total solution, } \\
V_{\text {STD_solution }}= & \text { volume of diluted standard solu- } \\
& \text { tion in vial, and } \\
V_{\text {headspace }}= & \text { volume of headspace. }
\end{array}
$$

6. Plot $\log [\mathrm{PFT}(\mathrm{g})]$ against $\log [$ peak area] and perform regression analysis to determine the calibration sensitivity. See Appendix B.

\section{Appendix B}

\section{Gas chromatograph calibration sensitivity for PFT analysis}

1. Perform the procedures for standard dilutions, injections, and peak integrations as described in Appendix A. Record the injection volume, dilution used, and peak area for each standard from a given site, organized by date of sample analysis.

2. For each site, plot the log of the PFT(g) contained in each injection against the log of the standard's integrated peak area (log[PFT injected] vs. $\log [$ peak area]). If necessary, discriminate between standards run on different dates by plotting them as separate series.

3. For each separate date of standard analysis (each series), add a linear trend line and display all corresponding equations.

4. From the trend line equations of the following format, record each series' $m$ and $b$ values:

$$
y=m x+b,
$$

where

$$
\begin{aligned}
y= & \log \text { of peak area, } \\
x= & \log \text { of mass injected, } \\
b= & \text { intercept derived from the calibration } \\
& \text { curve, and } \\
m= & \text { slope derived from the calibration curve. }
\end{aligned}
$$

5. Average the $m$ and $b$ values for each site. This implies that peak area $=10^{b}$ (PFT mass injected $)^{m}$.

6 . Use the average $m$ and $b$ values from each site to form the final calibration trend line (insert them into the generalized linear equation of $y=m x+$ b). 


\section{Appendix C}

\section{Gas chromatograph detection limit analysis}

1. Using a heated $3 \mathrm{~mL}$ gas-tight plastic syringe, perform $2 \mathrm{~mL}$ injections of outside laboratory air (blanks) both at the start and end of each day's sample analysis. Also run a blank injection immediately following any sample that produces an unusually high peak area. Expedition 329 blank vials containing ambient coring air are also run throughout analysis.

2. Compile a list of all blank peak areas produced from injections of ambient coring air run throughout Expedition 329 sample analysis.

3 . Calculate the average blank peak area from this compilation and use this value for the blank correction of measured peak areas.

4. Calculate the standard deviation of all blank peak areas.

5. The detection limit for blank corrected samples equals three standard deviations of all blank peak areas.

\section{Appendix D}

\section{Method for calculating the amount of drill water contamination}

The following equation implies that peak area $=10^{b}$ (PFT mass injected $)^{m}$ :

$$
\begin{gathered}
\text { drill water }(\mathrm{L}) / \text { core material }(\mathrm{g})= \\
{\left[\left(P_{\mathrm{BC}} / 10^{b}\right)^{1 / m}\right] /\left(C_{\mathrm{DW}} \times W \times F_{1}\right),}
\end{gathered}
$$

where

$P_{\mathrm{BC}}=$ blank corrected integrated peak area of PFT in injected sample (in arbitrary units),

$b$ = intercept derived from the calibration curve,

$m$ = slope derived from the calibration curve,

$C_{\mathrm{DW}}=\mathrm{PFT}$ concentration in drilling fluid (in grams/liter),

$W$ = weight of sediment sample (in grams), and

$F_{1}=$ fraction of total headspace injected:

$$
F_{1}=V_{\text {inj }} /\left[V_{\text {vial }}-\left(W / \rho_{\text {bulk }}\right)\right],
$$

where

$V_{\text {inj }}=$ volume of sample injected (in liters),

$V_{\text {vial }}=$ volume of vial (in liters),

$\rho_{\text {bulk }}=$ sediment sample density (in grams/liter), and

$W=$ weight of sample (in grams). 